\title{
A generalized view on normal moveout
}

\author{
Benjamin Schwarz* and Dirk Gajewski ${ }^{\dagger}$ \\ *University of Oxford, Department of Earth Sciences, \\ South Parks Road, Oxford OX1 3AN, United Kingdom \\ ${ }^{\dagger}$ University of Hamburg, Institute of Geophysics, \\ Bundesstr. 55, 20146 Hamburg, Germany
}

(June 4, 2017)

Running head: A generalized view on NMO

\begin{abstract}
While in the past, in the context of stacking, traveltime moveout was only formulated in individual common-midpoint (CMP) gathers, multi-parameter stacking utilizes normal moveout (NMO) approximations, which span several neighboring CMPs. Multi-parameter expressions such as the common reflection surface (CRS) or multifocusing are parameterized in terms of local slopes and curvatures of emerging wavefronts rather than effective velocities, which makes these approaches appear conceptually different from conventional velocity analysis. As a consequence, the unifying nature of multi-parameter NMO is still not well appreciated. In addition, CRS and multifocusing show distinctly different behavior in that they respond differently to both, overburden heterogeneity and curvature of the target interface, and either are or are not susceptible to moveout stretch. In this work we seek to demystify the wavefront picture by demonstrating that the conventional and multi-dimensional NMO operators can conveniently be derived from the same auxiliary straight-ray geometry, either representing the optical projection or formulated in an
\end{abstract}


effective replacement medium. Following the early work of de Bazelaire, we suggest a simple transformation between both domains and introduce generalized dual representations of the hyperbolic CRS, multifocusing, and the two recently introduced double-square-root expressions implicit CRS and nonhyperbolic CRS. In addition, we discuss a generalized finite-offset NMO expression that can likewise be applied to active-source diffraction data and passive seismic events. Synthetic examples suggest unification, conveniently explain the origin of moveout stretch and show that the joint use of different NMO approximations offers new insight into the character and origin of different wavefield components. 


\section{INTRODUCTION}

Normal moveout (NMO) still is a central ingredient in many existing processing workflows. In particular in stacking the knowledge of the trace-to-trace traveltime differences observed for locally coherent arrivals is essential to prevent undesired destructive interference during summation (Mayne, 1962). While it is generally possible to efficiently model moveout using e. g. the ray method, moveout itself is a collective property of locally coherent events and was shown to be measurable using trace-by-trace or group correlations and stacking schemes.

As a generalization of the classical CMP stack by Mayne (1962), the common-reflectionsurface (CRS) stack (Jäger et al., 2001) makes optimal use of the redundancy of information in the acquired data. Although a parabolic traveltime formula, which can be seen as a truncated Taylor series expansion of the traveltime, seems to be the most intuitive choice, numerical investigations as well as applications to synthetic and field data have suggested that a hyperbolic operator usually results in a better fit (e. g., Ursin, 1982; Schleicher et al., 1993). Because more complex settings are nowadays encountered in hydrocarbon exploration, diffractions and other highly curved subsurface features become increasingly important. In addition, due to the respective high angle coverage, diffractions are thought to contain super-resolved information about the subsurface (Khaidukov et al., 2004) and are strong indicators of fault structures. In addition they provide superior overburden illumination, which can be utilized e.g. in velocity model building (Bauer et al., 2016, 2017).

Fomel and Grechka (2001) concluded that even for a homogeneous background, the description of diffracted traveltimes is a higher-order problem and, in non-local vicinities, cannot be accurately governed by hyperbolic or parabolic operators, which can be derived from paraxial ray theory. Due to this nonhyperbolicity of diffractions and traveltimes from 
highly curved interfaces, efforts have been made to derive higher-order traveltime approximations (e.g., Landa et al., 2010; Fomel and Kazinnik, 2013; Schwarz et al., 2014) using the higher-order assumption of locally circular wavefronts. All of these traveltime approximations have in common that they can be derived from geometry, namely by assuming an analytical reflector shape and a constant velocity overburden. Inspired by the work of de Bazelaire (1988) and Höcht et al. (1999), Schwarz and Gajewski (2017) found that all non-parabolic operators can in fact be described in two types of auxiliary constant velocity media, which account for heterogeneity either by adapting the velocity of an effective overburden or by shifting the reference time to describe the problem's optical projection. They suggested a simple recipe to transform an effective medium operator, like the classical NMO hyperbola (Mayne, 1962), to its time-shifted counterpart in the optical domain and vice versa. As a result, the often misleading comparison of the hyperbolic CRS formula and the double-square-root multifocusing expression (Gelchinsky et al., 1999a,b) gets a clear explanation. In the frame of conflicting dip processing and global optimization by differential evolution, Walda et al. (2017) competetively compared the computational performance and attribute accuracy of different higher-order operators and commented on convergence and computational feasibility.

In this work, we review the geometrical intuitive picture of locally circular wavefronts (e.g. Shah, 1973; de Bazelaire, 1988; Höcht et al., 1999; Gelchinsky et al., 1999a,b) and introduce dual moveout expressions for active and passive seismic applications, where the auxiliary medium can be chosen by the user by supplying the according substitutions. Supported by a complex synthetic data example, we find that the double-square-root operators, when being expressed in the same auxiliary medium, can be considered equivalent NMO descriptions. Furthermore, we suggest the joint use of multiple NMO approximations to gain 
additional insight into the nature and origin of different wavefield components and explicitly illustrate the approaches practicability for the design of more discriminative diffraction filters. Finally, by means of synthetic examples, we provide an intuitive explanation of the undesired phenomenon of moveout stretch, which turns out to only appear for the effectivemedium NMO representations and briefly discuss strategies for passive seismic applications.

\section{CONVENTIONAL NMO}

[Figure 1 about here.]

The conventional normal moveout (NMO) hyperbola has its roots in the early 1960s (Mayne, 1962) and is still widely used in seismic exploration. For a planar horizontal reflector with homogeneous overburden it provides exact traveltimes and for dominantly horizontal layering, often encountered in sedimentary environments, it likewise is known to lead to accurate multi-coverage reflection traveltime estimates (e.g. Ursin, 1982). However, while for a constant velocity background, the constant velocity $v$ coincides with the actual medium velocity, in the general heterogeneous case it needs to be replaced with a constant effective velocity, i. e., a velocity shift $\delta v$ has to be applied. As can be inferred from Figure 1(a), the slope of the NMO hyperbola's asymptote is inversely proportional to the actual velocity in the homogeneous case or to the shifted (effective) velocity $v_{\text {shift }}=v+\delta v$, when the overburden is heterogeneous. Accordingly, the slope of the asymptote changes when the medium is heterogeneous and the hyperbolic moveout can be written as

$$
\Delta t\left(t_{0}, v_{\text {shift }}\right)=\sqrt{t_{0}^{2}+\frac{4 h^{2}}{v_{\text {shift }}^{2}}}-t_{0},
$$

where $t_{0}$ is the zero-offset two-way traveltime, which generally does not relate to the actual reflector depth (compare Figure 3). The adaption of $v_{\text {shift }}$ to account for heterogeneity 
forms the heart of the concept of velocity spectra and conventional velocity analysis (Taner and Koehler, 1969). In the late 80s, following the lines of Malovichko (1978), de Bazelaire (1988) approached NMO from a different viewpoint. Rather than adapting the overburden velocity, i.e., the slope of the hyperbola's asymptote, he suggested to shift the reference time $t_{\text {shift }}=t_{0}+\delta t$, which corresponds to a constant shift of the asymptote (see Figure 1(b)) to account for heterogeneity. His time-shifted hyperbolic NMO

$$
\Delta t\left(t_{\text {shift }}, v_{0}\right)=\sqrt{t_{\text {shift }}^{2}+\frac{4 h^{2}}{v_{0}^{2}}}-t_{\text {shift }}
$$

in contrast to the conventional NMO hyperbola (1) does not depend on the actual reference time $t_{0}$ but for a constant near-surface velocity $v_{0}$ remains a one-parameter equation. Similar to conventional velocity analysis, a coherence-driven scan for the fit-optimizing $t_{\text {shift }}$ leads to automatable NMO correction and likewise permits Dix-type interval velocity inversion (Hubral et al., 1980; de Bazelaire, 1988; Castle, 1994). De Bazelaire, in contrast to other authors was the first to emphasize the strong connection of the shifted hyperbola to optics, as he provided an intuitive geometrical derivation for the auxiliary straight-ray analog of the optical projection of the reflection process. Both approaches, a shift in velocity and a shift in time lead to a comparable adaption of the moveout and therefore represent two varieties of the same hyperbolic CMP moveout. In the following, we review the concept of multi-parameter NMO and the wavefront picture, which permits us to intuitively discuss current non-hyperbolic multi-parameter NMO expressions under the same conceptual roof.

\section{MULTI-PARAMETER NMO AND THE WAVEFRONT PICTURE}

[Figure 2 about here.]

While prestack depth imaging methods seek to describe the full wavefield with a single 
global model of the subsurface, time imaging methods like stacking do not aim at describing the global, but the local wavefield with operators, which are only valid in a certain small vicinity of the imaging point. Without loss of generality, one can assume that, as long as we confine ourselves to a reasonably small vicinity of the zero-offset recording location $x_{0}$, the prestack traveltimes of neighboring rays can be described by the truncated Taylor series expansion,

$$
t\left(\Delta x_{m}, h\right) \approx t_{0}+a \Delta x_{m}+b \Delta x_{m}^{2}+c h^{2}
$$

where $\Delta x_{m}=x_{m}-x_{0}$ and $h$ denote midpoint separation and half-offset between source and receiver, respectively. The zero-order term $t_{0}$ is the traveltime of the normal ray observed at coordinate $x_{0}$. The first- and second-order coefficients $a, b$, and $c$ represent slope and curvatures of the traveltime surface $t\left(\Delta x_{m}, h\right)$ recorded in the vicinity of $x_{0}$ (see, e.g., Schleicher et al., 1993).

In the high-frequency approximation, the concepts of rays and wavefronts are equally important (Červený, 2001). While the rays can be considered integral quantities, containing all their past, a wavefront can be interpreted as a snapshot showing the end positions of infinitely many neighboring rays corresponding to the same propagation time. Consequently, the ray concept is well-suited to describe total traveltimes, whereas a set of wavefronts helps to compare the traveltimes at two spatially separated positions. While equation (3) was originally derived from paraxial ray theory (e.g. Bortfeld, 1989), we propose to view moveout from the perspective of wavefronts, which allow simple geometrical derivations of expressions of higher order than two. In fact, Hubral (1983) showed that the coefficients of the second-order expression (3) can be related to the local properties of two conceptual one-way wavefronts, representing a fictitious exploding point source and an exploding re- 
flector experiment located at the normal incidence point (NIP) on the imaged reflector (see Figure 2) via

$$
\begin{gathered}
a=\left.\frac{\partial t}{\partial x_{m}}\right|_{\left(x_{m}=x_{0}, h=0\right)}=\frac{2 \sin \alpha}{v_{0}}, \\
b=\left.\frac{1}{2} \frac{\partial^{2} t}{\partial x_{m}^{2}}\right|_{\left(x_{m}=x_{0}, h=0\right)}=\frac{\cos ^{2} \alpha}{v_{0} R_{\mathrm{N}}}, \\
c=\left.\frac{1}{2} \frac{\partial^{2} t}{\partial h^{2}}\right|_{\left(x_{m}=x_{0}, h=0\right)}=\frac{\cos ^{2} \alpha}{v_{0} R_{\mathrm{NIP}}} .
\end{gathered}
$$

According to these expressions, in connection with the parabolic formula (3), the traveltime of the reference ray $t_{0}$ has no influence on the traveltime differences following from the inclination and curvature of the conceptual wavefronts, i.e. the traveltime moveout, in the frame of this approximation, solely depends on the first- and second-order attributes (Hubral, 1983),

$$
t-t_{0}=\Delta t\left(\alpha, R_{\mathrm{NIP}}, R_{\mathrm{N}}\right) \quad .
$$

The common-reflection-surface (CRS) stack by Jäger et al. (2001) can be viewed as a generalization of the classical CMP stack to neighboring CMPs. As a consequence, as can also be inferred from equations (4a) to (4c), the number of degrees of freedom to fit the moveout surface to the data increases from one to three. By taking several adjacent CMP gathers into account, the contribution of dip moveout (DMO, e.g. Deregowski, 1986) is separable from heterogeneity effects and wavefront tomography, utilizing the estimated wavefront attributes, allows for laterally resolved velocity inversion beyond Dix (e.g. Duveneck, 2004; Hertweck et al., 2007).

[Figure 3 about here.] 


\section{Locally circular wavefronts}

[Figure 4 about here.]

To make maximum use of information redundancy in the data, stacking methods seek to include higher offsets, which, in principle implies a need for additional higher-order terms in the traveltime operator (3). Since a higher dimensionality of the traveltime fit may lead to instabilities and to undesired additional computational costs, several non-parabolic approximations have been introduced (e.g., Landa et al., 2010; Fomel and Kazinnik, 2013; Schwarz et al., 2014), which all have in common that the number of degrees of freedom is not increased. In the following, we will show that, provided lateral velocity changes are small, these higher-order moveout approximations can account for heterogeneity by two different mechanisms, both relying on the assumption of an auxiliary medium of constant velocity. Although sometimes viewed as competing concepts, rays and wavefronts are generally coupled to each other. In isotropic media, rays are always normal on the wavefront (e.g. Červený, 2001) and for the homogeneous case, the length of a ray $R_{0}$ is proportional to its traveltime $t_{0}=R_{0} / v$ and exactly equal to the curvature radius of the respective wavefront. At a later time $t=t_{0}+\Delta t$, the corresponding wavefront radius reads

$$
R=R_{0}+v \Delta t
$$

which lets us arrive at the following simple expression for the traveltime moveout

$$
\Delta t=\frac{R-R_{0}}{v}
$$

where the radius of curvature $R$ of the finite-offset wavefront measurement can be expressed in terms of the attributes of the reference wavefront $\alpha, R_{0}$, and the lateral offset $\Delta x$ (compare 
Figure 4),

$$
R=\sqrt{R_{0}^{2}+2 R_{0} \Delta x \sin \alpha+\Delta x^{2}}
$$

As we will demonstrate in the following, formula (7) together with the circular wavefront definition (8), despite their formal simplicity, can be used to derive all well-established closed-form isotropic moveout expressions, including the hyperbolic CRS formula (Jäger et al., 2001), multifocusing (Gelchinsky et al., 1999a,b), the implicit CRS (Schwarz et al., 2014) and the nonhyperbolic CRS approximation introduced by Fomel and Kazinnik (2013). Although the underlying assumption of homogeneity is, of course, generally not fulfilled, we show how the concept of circular wavefronts and, consequently, straight rays can also be maintained for the heterogeneous case (see also Schwarz and Gajewski, 2017). While the utilized principal ambiguity of surface-based wavefront measurements can complicate the inversion of subsurface properties in depth, it likewise allows for the replacement of a rather complicated ray geometry by a simple one-way problem, which is much easier and more convenient to describe.

\section{HETEROGENEITY}

Having been introduced in the context of migration (Hubral, 1983), the wavefront attributes $\alpha, R_{\mathrm{NIP}}$, and $R_{\mathrm{N}}$ contain both geometrical properties of the imaged reflector and propagation effects due to the overburden. According to the NIP-wave theorem (Hubral, 1983), hyperbolic moveout with half-offset $h$, i. e., in the CMP gather, does not depend on the curvature of the reflector, which is why curvature is not accounted for in formulae (1) and (2). In midpoint direction, however, reflector curvature is a lower, i. e., second-order effect

and generally has a strong impact on the traveltime differences of neighboring rays (see 
equations (3) and (4b)).

In the following, we suggest an alternative to the conventional wavefront parameterization by making use of the fact that certain combinations of the parameters remain unaffected by the choice of the auxiliary medium. While in conventional CRS the reflector curvature is related to the parameter $R_{\mathrm{N}}$, we now suggest to instead use the combination

$$
\rho=\frac{R_{\mathrm{NIP}}}{R_{\mathrm{N}}},
$$

since it remains unchanged, when we go from the constant near-surface velocity medium of the time shift to the effective constant velocity medium underlying the approach of shifting the velocity and vice versa. In addition, the horizontal slowness or ray parameter

$$
p=\frac{a}{2}=\frac{\sin \alpha}{v_{0}},
$$

according to equation (4a) and following from intuition, should be independent of the choice of the auxiliary medium, since it describes the midpoint (i.e. zero-offset) slope of the actual reflection event in the vicinity of the central measurement location $x_{0}$. Due to the fact that both the first-order parameter $p$ and the dimensionless parameter $\rho$ are unaffected by the choice of the auxiliary medium, they can be considered propagation invariants. The shifted zero-offset traveltime represents the reference time of the projected problem and therefore is defined in the auxiliary medium of constant near-surface velocity. As can be concluded from geometry (see Figure 3), it bears a close connection to the NIP wave radius,

$$
t_{\mathrm{shift}}=\frac{2 R_{\mathrm{NIP}}}{v_{0}}
$$

and, as a consequence, following from this geometrical reasoning, links the zero-order and the second-order terms of expansion (3). The relationship of the shifted velocity to the CRS attributes was already established, e. g. by Mann (2002) by identifying it in the reduced hyperbolic CRS formula for diffractions, or independently by Schwarz et al. (2014) 
by matching the expansion coefficients of the suggested geometrical implicit CRS approach to the coefficients (4a) to (4c). Following the choice of the horizontal slowness as an independent parameter, this velocity can be defined in slowness notation, which seems more consistent and turns out to simplify expressions

$$
\frac{1}{v_{\text {shift }}^{2}}=p^{2}+\frac{t_{0}}{t_{\text {shift }}}\left(\frac{1}{v_{0}^{2}}-p^{2}\right) \quad .
$$

Since in this formula, the shifted velocity is linked to the time shift of the projection approach, and, in accordance with the early work by de Bazelaire (1988), who motivated the use of concepts from geometrical optics, we consider it a generalized osculating equation, which is also valid in the frame of multi-parameter NMO (compare Figure 5(a) and (b)). In the following, we parameterize the hyperbolic CRS formula (e. g., Jäger et al., 2001), the nonhyperbolic implicit CRS (Schwarz et al., 2014), and multifocusing (Gelchinsky et al., 1999a,b) by means of the above suggested substitutions (9) to (12).

\section{Auxiliary media and the NIP wave}

By closer investigating the formal structure of the generalized parameterization introduced previously, it becomes obvious that the two mechanisms suggested to account for heterogeneity are both connected to properties of the NIP wave only. In the context of true-amplitude migration, Hubral (1983) introduced the NIP concept, in which a fictitious wave is thought to be initiated at the normal incidence point (see Figure 2). Based on the fundamental assumptions of ray path symmetry and reciprocity, this NIP wave is initiated at zero time, travels at half the actual medium velocity, and finally is measured at the central zero-offset location $x_{0}$ (compare again Figure 2 ) at time $t_{0}$, which is equal to the true zero-offset refer-

ence ray traveltime. So in conclusion, the NIP wave (in the circular wavefront picture) can 
be characterized via the full set of properties

$$
\left(t_{0}, \alpha, R_{\mathrm{NIP}}\right)
$$

measured at the surface. Due to the fact that this wavefront, according to its definition is known to have infinite curvature and is multidirectional when initiated at the NIP, and since the traveltime is equal to the actual zero-offset reference traveltime, the NIP wave, in principle contains all heterogeneity effects felt in the overburden during the expiration of time $t_{0}$. Bearing in mind that, consequently, only the full set (13) fully characterizes the NIP wave measurement, the two different suggested mechanisms, consisting either in a shift in time or a shift in velocity, can be pictured very intuitively. In essence, both approaches try to approximate the actual NIP experiment from two different views. Being confined by the straight-ray assumption, they can only accurately describe certain aspects of the NIP wave, i.e. a subset of (13).

According to de Bazelaire (1988) and Höcht et al. (1999), the time shift mechanism maintains the straight-ray (or circular wavefront) assumption by shifting the actual reference time $t_{0}$, which, as argued above, is also the actual traveltime of the NIP wave, to the value (11), which corresponds to the optical straight-ray projection analog of the actual wavefront measurement. Thus, the time-shift mechanism accurately describes the geometry of the surface recording of the actual NIP wavefront and the corresponding emergence angle, but fails at physically describing the actual traveltime of the NIP wave, denoted by $t_{0}$, due to the imposed straight-ray limitation. In consequence, the time-shift approaches do not incorporate the actual reference time $t_{0}$, but only use the actual measured wavefront curvature and angles to approximate traveltime differences. If a velocity-shift is consid- 
ered to account for heterogeneity, the constant velocity is adapted to the actual NIP wave traveltime, which corresponds to the true recorded zero-offset reference traveltime $t_{0}$. As a consequence, the other surface-based NIP wave attributes, i. e. the actual curvature radius $R_{\mathrm{NIP}}$ and the emergence angle $\alpha$ get perturbed by this velocity change and, again, due to the straight-ray limitations, do not represent the actual surface measurements anymore. They rather are represented by effective properties, which, due to the generalized osculating equation, can be related to their actual values via (Mann, 2002; Schwarz et al., 2014)

$$
\begin{aligned}
\alpha_{\mathrm{eff}} & =\arcsin \left(\frac{v_{\mathrm{shift}}}{v_{0}} \sin \alpha\right), \\
R_{\mathrm{NIP}}^{\mathrm{eff}} & =\frac{v_{\text {shift }} t_{0}}{2}
\end{aligned}
$$

Note that the system (14) can be inserted into any CRS-type operator (like, e.g., conventional multifocusing) to arrive at its effective medium representation. If the considered operator is already formulated in the effective medium, it will not change under the above substitutions.

\section{GENERALIZED NMO EXPRESSIONS}

[Figure 5 about here.]

For the two different representations of the CMP hyperbola (1) and (2), it becomes immediately obvious that a simple exchange $\left(t_{0}, v_{\text {shift }}\right) \leftrightarrow\left(t_{\text {shift }}, v_{0}\right)$ transforms the initial operator to the other representation. We conclude from this fact that each moveout expression can be written in a generalized form, i. e., for a general auxiliary medium of constant velocity $\hat{v}$ with its respective reference time $\hat{t}_{0}$. Depending on the choice of $\left(\hat{t}_{0}, \hat{v}\right)$, this generalized moveout $\Delta \hat{t}$ has two faces, one accounting for heterogeneity by means of a time shift, 
the other making use of an effective overburden velocity (Schwarz and Gajewski, 2017). We would like to emphasize in this context that the choice of either the effective or the near-surface velocity for the auxiliary medium directly implies the corresponding reference traveltime. This connection is expressed by the generalized osculating equation (12), which can be rewritten as

$$
\frac{1}{\hat{v}^{2}}=p^{2}+\frac{\hat{t}_{0}}{t_{\text {shift }}}\left(\frac{1}{v_{0}^{2}}-p^{2}\right) .
$$

As will be supported by data examples, the dependence of moveout on the actual reference traveltime $t_{0}$ has striking implications, like the effect of moveout stretch after NMO correction or the potential for the estimation of the excitation time of a passive seismic source. Following from equations (14), the attributes of the NIP wave can likewise be written in a generalized form,

$$
\begin{aligned}
\hat{\alpha} & =\arcsin \left(\frac{\hat{v}}{v_{0}} \sin \alpha\right), \\
\hat{R}_{\mathrm{NIP}} & =\frac{\hat{v} \hat{t}_{0}}{2} .
\end{aligned}
$$

Depending on the choice of the auxiliary medium, the above emergence angle and curvature radius of the NIP wave either correpond to the true surface measurements, or they represent effective properties. In the following, we introduce generalized versions of the hyperbolic CRS, implicit CRS (iCRS), nonhyperbolic CRS, and multifocusing operators and reveal in which auxiliary medium they were originally formulated.

It is well known that double-square-root type approximations are better suited for the accurate description of traveltime moveout for reasonably curved target discontinuities (e.g. Fomel and Grechka, 2001). It can be inferred from equation (5) that in the presented frame of locally circular wavefronts, the attributes of two waves are needed to describe the moveout arising from the scattering at tilted and curved subsurface structures. In accordance, in the 
most general sense, traveltime differences observed for a paraxial source in the vicinity of a reference source $(i=s)$ and a paraxial receiver in the local vicinity of a respective reference receiver $(i=g)$ can be written as

$$
\Delta \hat{t}=\sum_{i=s, g} \frac{\hat{R}_{i}-\hat{R}_{0 i}}{\hat{v}_{i}}
$$

where $R_{i}$ and $R_{0 i}=R_{i}\left(\Delta x_{m}=0, h=0\right)$ denote the paraxial and the reference wavefront curvature radii, respectively. In this work, we mainly investigate normal moveout, i. e. moveout with respect to a zero-offset reference ray, where $\hat{v}_{s}=\hat{v}_{g}=\hat{v}$. Nevertheless, equation (17) is likewise applicable to the more general finite-offset case (see, e.g., Berkovitch et al., 2011).

\section{Hyperbolic moveout}

The CRS stack is a natural generalization of the CMP stack, in which redundant information from neighboring CMP gathers is exploited in the stacking process. Due to this incorporation of adjacent CMPs, the according moveout is a surface rather than a curve. Although the parabolic formula (3), being equivalent to the a truncated second-order Taylor series expansion of the traveltime, seems the most natural choice, most moveout investigations appearing in literature are based on a hyperbolic expression (Ursin, 1982; Bortfeld, 1989; Schleicher et al., 1993). For the hyperbolic approximation, which is valid for the case of an inclined but planar target reflector (Levin, 1971), source and receiver contributions to the moveout are considered to be symmetric, i.e. we have $\hat{R}_{s}=\hat{R}_{g}=\hat{R}$ and $\hat{R}_{0 s}=\hat{R}_{0 g}=\hat{R}_{\mathrm{NIP}}$ and the paraxial wavefront radius can be approximated as (e.g. Höcht et al., 1999)

$$
\hat{R} \approx \sqrt{\left(\hat{R}_{\mathrm{NIP}}+\sin \hat{\alpha} \Delta x_{m}\right)^{2}+\cos ^{2} \hat{\alpha}\left(\rho \Delta x_{m}^{2}+h^{2}\right)} .
$$


Similar to the classical CMP stack, numerical investigations as well as application in actual sedimentary environments (e. g. Ursin, 1982; Jäger et al., 2001) support the notion that the hyperbolic CRS moveout is more suited than the parabolic formula when the subsurface consists of a mostly horizontally layered system. Although it has been introduced via squaring the parabolic expression (3) (e.g. Bortfeld, 1989; Schleicher et al., 1993), gained from paraxial ray theory (with subsequent neglection of terms of higher order than two), expression (18) can also be derived from geometry (see, e.g., Höcht et al., 1999). For confinement to the CMP gather, i.e. $\Delta x_{m}=0$, the generalized hyperbolic CRS expression reduces to the generalized $\mathrm{CMP}$ moveout,

$$
\hat{R}=\sqrt{\hat{R}_{\mathrm{NIP}}^{2}+\cos ^{2} \hat{\alpha} h^{2}},
$$

which, depending on the choice of reference time $\hat{t}_{0}$ and velocity $\hat{v}$ and, in consequence, the auxiliary medium, reduces either to formula (1) or (2) for horizontal layering. It is interesting to note that, similar to the nonhyperbolic higher-dimensional moveouts discussed in the following, the hyperbolic CRS formula (18) is exact for a certain subsurface model, which in this case constitutes of a planar dipping target reflector beneath a constant velocity overburden (Schleicher et al., 1993). Both formulae, the hyperbolic CRS expression and the classical CMP hyperbola were first formulated in the effective constant velocity auxiliary medium, which is why their moveout correction depends on the reference time $t_{0}$ and therefore also may result in wavelet stretch after correction. While the second face of the CMP hyperbola was already suggested by de Bazelaire in the late eighties (de Bazelaire, 1988), the time-shifted version of hyperbolic CRS was briefly mentioned only in the work of Höcht et al. (1999) and now finds its solid theoretical foundation in the context of the auxiliary media interpretation of higher-order NMO. 


\section{Multifocusing}

Coexisting with the hyperbolic CRS method for over fifteen years, the multifocusing approach (Gelchinsky et al., 1999a,b) is based on a double-square-root NMO expression. Similar to the hyperbolic CRS formula (18), this moveout can be derived from geometry and therefore intrinsically relies on the assumption of straight rays (e.g., Landa et al., 2010). Due to the higher mathematical complexity, the multifocusing moveout has not gained sufficient attention for a long time (e. g. Tygel et al., 1999), but became increasingly important to the community with the rise of interest in imaging diffracted seismic events (Landa et al., 2010). Although it is based on the same wavefront attributes as the CRS method, namely $\alpha, R_{\mathrm{NIP}}$, and $R_{\mathrm{N}}$, it turns out to noticeably deviate from the hyperbolic approximation when reflectors are curved and when the overburden is heterogeneous (e. g. Schwarz et al., 2014). Multifocusing was originally derived in the wavefront picture and, therefore, is naturally expressed by the generalized moveout expression (17). It is formulated in terms of a so-called focusing-point (FP) wave emitted by a fictitious point source located at the intersection point of the zero-offset reference and the considered paraxial ray (see Figure 6(a)). The corresponding curvature radii, in perfect agreement with equation (8), read

$$
\hat{R}_{i}=\sqrt{\hat{R}_{0 i}^{2}+2 \hat{R}_{0 i} \Delta x_{i} \sin \hat{\alpha}+\Delta x_{i}^{2}}
$$

with $i=s, g$. Similar to the paraxial source and receiver radii (20), the reference wavefront curvature radii can be identified in the FP wave straight-ray geometry in Figure 6(a) and are related to the attributes of the NIP and the normal wave via

$$
\begin{aligned}
\hat{R}_{0 s} & =\frac{1-\hat{\sigma}}{\rho-\hat{\sigma}} \hat{R}_{\mathrm{NIP}}, \\
\hat{R}_{0 g} & =\frac{1+\hat{\sigma}}{\rho+\hat{\sigma}} \hat{R}_{\mathrm{NIP}},
\end{aligned}
$$


where the so-called focusing parameter $\hat{\sigma}$ is a function of the source and receiver offsets $\Delta x_{s}=x_{s}-x_{0}$ and $\Delta x_{g}=x_{g}-x_{0}$ and therefore changes for each individual ray considered in the vicinity of the central ray emerging at midpoint $x_{0}$. In the most commonly used planar approximation (Gelchinsky et al., 1999a,b; Landa et al., 2010), it reads

$$
\hat{\sigma}=\frac{\Delta x_{g}-\Delta x_{s}}{\Delta x_{s}+\Delta x_{g}+2 \hat{R}_{\mathrm{NIP}}^{-1} \Delta x_{s} \Delta x_{g} \sin \hat{\alpha}}
$$

Please observe that, in contrast to the hyperbolic CRS approach and its classical CMP hyperbola subset, which almost exclusively appear as effective medium representations in literature (Schwarz and Gajewski, 2017), multifocusing originally describes the projected problem observed in the constant near-surface velocity medium. Therefore, it appears in literature in time-shift parameterization. So for the choice of the effective medium, i. e. for $\left(\hat{t}_{0}, \hat{v}\right)=\left(t_{0}, v_{\text {shift }}\right)$, we arrive at a novel multifocusing moveout approximation.

[Figure 6 about here.]

\section{Implicit common reflection surface}

Starting from geometrical considerations, Schwarz and Gajewski (2017) observed that the nonhyperbolic double-square-root type implicit CRS operator can be represented not only in one but two auxiliary domains. While the velocity-shifted effective medium representation turned out to behave similarly to hyperbolic CRS for moderate reflector curvatures and mostly vertical velocity changes in the overburden, the time-shifted version showed a strikingly strong resemblance to multifocusing, theoretically, and backed up by data examples, for the case of high reflector curvatures and in the diffraction limit. Similar to the multifocusing approach, the implicit CRS treats the down- and up-going contributions of the approximated raypaths separately. The circular wavefront radii approximations in the 
generalized auxiliary medium of constant velocity $\hat{v}$ again can be derived from simple geometry and correspond to paraxial measurements of a wavefront originating from a fictitious point source located at the reflection point (RP, compare Figure 6(b))

$$
\hat{R}_{i}=\sqrt{\left(\Delta x_{i}-\hat{x}_{\mathrm{RP}}\right)^{2}+\hat{z}_{\mathrm{RP}}^{2}}
$$

where $i=s, g$ and

$$
\begin{aligned}
& \hat{x}_{\mathrm{RP}}=\frac{\sin \hat{\alpha}+(\rho-1) \sin \hat{\theta}}{\rho} \hat{R}_{\mathrm{NIP}}, \\
& \hat{z}_{\mathrm{RP}}=\frac{\cos \hat{\alpha}+(\rho-1) \cos \hat{\theta}}{\rho} \hat{R}_{\mathrm{NIP}}
\end{aligned}
$$

denote the lateral position and depth of the reflection point on the locally circular interface relative to the central midpoint location $\left(x_{0}, 0\right)$. The components, similar to the radii of the source and and receiver focusing wavefront attributes (21) change with every paraxial source-receiver combination and are linked via the geometrically derived quantity

$$
\tan \hat{\theta}=\tan \hat{\alpha}+\frac{\rho \Delta x_{m}}{\hat{R}_{\mathrm{NIP}} \cos \hat{\alpha}}+\frac{\rho h}{\hat{R}_{\mathrm{NIP}} \cos \hat{\alpha}} \frac{\hat{R}_{s}-\hat{R}_{g}}{\hat{R}_{s}+\hat{R}_{g}}
$$

Please note that this contribution does not enter (24) for the diffraction case, where $\rho=$ $\hat{R}_{\mathrm{NIP}} / \hat{R}_{\mathrm{N}}=1$. Solving the problem of reflection from a circular reflector in a constant velocity medium, implicit CRS iteratively refines the angle $\theta$, which geometrically represents the angle defining the finite-offset reflection point. It therefore plays a similar role as the focusing parameter (22) in connecting traveltime contributions of the up- and down-going ray segments. Like the hyperbolic CRS moveout and in contrast to multifocusing, the implicit CRS moveout was introduced in the effective medium. Therefore, the time-shifted (optical) version with $\left(\hat{t}_{0}, \hat{v}\right)=\left(t_{\text {shift }}, v_{0}\right)$ corresponds to a new alternative to the conventional implicit CRS moveout suggested by Schwarz et al. (2014). 


\section{Nonhyperbolic common reflection surface}

Fomel and Kazinnik (2013) introduced a higher-order alternative to the hyperbolic CRS moveout which, similar to multifocusing, can be written as a double-square-root equation. In contrast to the implicit CRS, their so-called nonhyperbolic CRS expression was not derived from the geometry of a circular, but of a vertically oriented hyperbolic reflector shape (Fomel and Stovas, 2010). Following from the geometry shown in Figure 6(c), the moveout can likewise be conveniently formulated in terms of local measurements of a wavefront originating from the finite-offset reflection point (RP). Therefore, equations (17) and (23) are equally valid for the generalized nonhyperbolic CRS approximation. Just like for the implicit CRS, we have $\hat{R}_{0 s}=\hat{R}_{0 g}=\hat{R}_{\mathrm{NIP}}$, i.e. the reference curvature radius is again the one of the NIP wave, viewed in the generalized auxiliary medium of constant velocity $\hat{v}$. Following the geometrical derivation of Fomel and Stovas (2010), the position of the reflection point $\left(\hat{x}_{\mathrm{RP}}, \hat{z}_{\mathrm{RP}}\right)$ can be written as

$$
\begin{aligned}
& \hat{x}_{\mathrm{RP}}=\frac{\hat{z}_{\text {hyp }}^{2}\left(\hat{x}_{s}+\hat{x}_{g}\right) \cos ^{2} \hat{\beta}}{\hat{z}_{\text {hyp }}^{2}-\hat{x}_{s} \hat{x}_{g} \sin ^{2} \hat{\beta}+\sqrt{\left(\hat{z}_{\text {hyp }}^{2}+\hat{x}_{s} \sin ^{2} \hat{\beta}\right)\left(\hat{z}_{\text {hyp }}^{2}+\hat{x}_{g} \sin ^{2} \hat{\beta}\right)}}, \\
& \hat{z}_{\mathrm{RP}}=\sqrt{\hat{z}_{\text {hyp }}^{2}+\hat{x}_{\mathrm{RP}}^{2} \tan ^{2} \hat{\beta}}
\end{aligned}
$$

where $\hat{x}_{s}=\hat{x}_{\mathrm{hyp}}+\Delta x_{m}-h$ and $\hat{x}_{g}=\hat{x}_{\mathrm{hyp}}+\Delta x_{m}+h$ are the paraxial source and receiver positions relative to the apex of the vertically oriented hyperbola (compare Figure 6(c)). The cooresponding coordinates

$$
\begin{aligned}
& \hat{x}_{\mathrm{hyp}}=\frac{\sin \hat{\alpha}}{\sin ^{2} \hat{\alpha}+\rho \cos ^{2} \hat{\alpha}} \hat{R}_{\mathrm{NIP}}, \\
& \hat{z}_{\mathrm{hyp}}=\sqrt{\frac{\rho}{\rho+\tan ^{2} \hat{\alpha}}} \hat{R}_{\mathrm{NIP}} .
\end{aligned}
$$


can be formulated in terms of the NIP wave attributes $\hat{\alpha}$ and $\hat{R}_{\mathrm{NIP}}$, which can also be used to express the tilt angle $\hat{\beta}$ of the hyperbolic reflector's asymptote via

$$
\tan \hat{\beta}=\sqrt{\frac{\tan ^{2} \hat{\alpha}+\rho}{1-\rho}} .
$$

Although at first sight, expressions (26) together with (27) and (28) seem more complicated than the implicit CRS counterparts, we need to stress that they are of explicit nature, whereas the implicit CRS requires iterative refinement of the reflection point coordinates (Schwarz et al., 2014). Thus, the use of a hyperbolic reflector allowed for a closed-form solution, but the connection to the NIP wave attributes $\hat{\alpha}$ and $\hat{R}_{\mathrm{NIP}}$ and the normalized curvature ratio $\rho$ is less intuitive than for the circular reflector shape. Similar to the implicit CRS, the nonhyperbolic CRS was originally introduced as an effective-medium operator, i.e. the choice of $\left(\hat{t}_{0}, \hat{v}\right)=\left(t_{0}, v_{\text {shift }}\right)$ leads to the result found by Fomel and Kazinnik (2013). Consequently, setting $\left(\hat{t}_{0}, \hat{v}\right)=\left(t_{\text {shift }}, v_{0}\right)$ results in a new alternative expression describing the optical projection in the auxiliary near-surface velocity medium.

\section{Common-reflection-point moveout}

The common-reflection-point (CRP) gather is an important data configuration, because in contrast to the conventional CMP gather it takes dip moveout (DMO) and, consequently, reflection point dispersal into account (e.g. Deregowski, 1986). In the wavefront picture, the common-reflection-point moveout, as can also be inferred from Figure 2, builds on reciprocity and can be fully understood by studying the one-way propagation of the NIP wave. In the circular wavefront approximation and for a zero-offset reference, we thus have $\hat{R}_{0 s}=\hat{R}_{0 g}=\hat{R}_{\mathrm{NIP}}$ and

$$
\hat{R}_{i}=\sqrt{\hat{R}_{\mathrm{NIP}}^{2}+2 \hat{R}_{\mathrm{NIP}} \Delta x_{i} \sin \hat{\alpha}+\Delta x_{i}^{2}}
$$


where $i=s, g$. Due to Snell's law, only certain source-receiver combinations $\Delta x_{s}=\Delta x_{s}\left(\Delta x_{g}\right)$ can be described by equation (29), which makes the concept considerably less practical than the conventional CMP approach. The implicit and the nonhyperbolic CRS moveouts discussed previously are particularly suited to study the moveout in the CRP gather, since they both evaluate the reflection point $\left(\hat{x}_{\mathrm{RP}}, \hat{z}_{\mathrm{RP}}\right)$ for each individual paraxial finite-offset. Accordingly, both approximations can be expressed in terms of a fictitious wavefront, originating at the reflection point (see Figure 6). Its geometrical tilt and curvature

$$
\begin{aligned}
& \hat{\alpha}_{\mathrm{RP}}=\arctan \left(\frac{\hat{x}_{\mathrm{RP}}}{\hat{z}_{\mathrm{RP}}}\right), \\
& \hat{R}_{\mathrm{RP}}=\sqrt{\hat{x}_{\mathrm{RP}}^{2}+\hat{z}_{\mathrm{RP}}^{2}},
\end{aligned}
$$

according to either equations (24) or (26) depend on the source and receiver separation from the central midpoint $\left(x_{0}, 0\right)$. For $\hat{\theta} \rightarrow \hat{\alpha}$ in equation (25) or for $\hat{\sigma}^{-1} \rightarrow 0$ (see equation (22)), the generalized expressions of the implicit CRS and multifocusing take the form of the generalized CRP wavefront curvature radii defined according to (29). Accordingly, these transitions lead to $\left(\hat{\alpha}_{\mathrm{RP}}, \hat{R}_{\mathrm{RP}}\right)$ and result in the same source-receiver coordinate coupling condition

$$
\Delta \hat{x}_{s}\left(\Delta x_{g}\right)=-\frac{\Delta x_{g}}{1+2 \hat{R}_{\mathrm{NIP}}^{-1} \sin \hat{\alpha} \Delta x_{g}} \quad,
$$

which was already found by other authors (e.g. Höcht et al., 1999; Perroud et al., 1999; Cruz et al., 2000), here formulated in the generalized auxiliary medium, which can either be effective or corresponds to the straight-ray domain of the optical projection. Insertion of expression (31) into the paraxial source and receiver measurements of the NIP wavefront (29) and switching to midpoint-half-offset coordinates leads to the same parametric expressions found by Höcht et al. (1999). For all double-square-root operators mentioned before, the CRP moveout is naturally incorporated as a special subset, which, together with their 
similarity (or even exact correspondence) in the zero-offset section suggests a high degree of unification.

\section{Diffractions and passive events}

[Figure 7 about here.]

[Figure 8 about here.]

When the reflector curvature approaches infinity with respect to the dominant seismic wavelength, the normalized propagation invariant $\rho$ approaches unity and the traveltime moveout is again, just like in the common-reflection-point gather, fully governed by the one-way propagation of the NIP wave. In contrast to the common-reflection-point scenario however, the previously found generalized coupling of the source and receiver coordinates (31) vanishes, which is directly related to the fact that Snell's law breaks down for infinitely curved back-scattering subsurface structures (see Figure 7). Due to this lack of coupling of the source and receiver moveout contributions, equation (17) can even be used to infer a higher-order finite-offset moveout approximation. Following the simple straight-ray geometry in Figure 4, we arrive at

$$
\hat{R}_{i}=\sqrt{\hat{R}_{0 i}^{2}+2 \hat{R}_{0 i} \Delta x_{i} \sin \hat{\alpha}_{i}+\Delta x_{i}^{2}}
$$

where $\Delta x_{i}=x_{i}-x_{0 i}$ for the source $(i=s)$ and receiver $(i=g)$ measurement, respectively. As can be observed in Figure 7, for the finite-offset case, moveout is determined with respect to the respective source and receiver segment of the reference ray, which in contrast to the zero-offset case generally do not coincide. Likewise in contrast to the zero-offset scenario, the diffraction moveout, from the circular wavefront perspective, is parameterized 
by attributes $\left(\hat{\alpha}_{i}, \hat{R}_{0 i}\right)$ of two separate wavefront measurements, one carried out at the reference source $(i=s)$, the other at the reference receiver location $(i=g)$. Similar to the other approximations, we suggest two dual versions of the finite-offset diffraction moveout, where

$$
\begin{aligned}
& \hat{\alpha}_{i}=\arcsin \left(\frac{\hat{v}_{i}}{v_{0 i}} \sin \alpha_{i}\right), \\
& \hat{R}_{0 i}=\hat{R}_{\mathrm{NIP}}^{i}=\hat{v}_{i} \hat{t}_{0 i} .
\end{aligned}
$$

Equations (33), in contrast to their zero-offset counterparts (16) correspond to attributes of true one-way processes, independently defined for the common-shot $(i=g)$ and the common-receiver $(i=s)$ configuration. In the shot gather, the quantities (33) parameterize the actual physical wavefront arriving at the receiver array (compare Figure 7). Accordingly, $t_{0 i}$ corresponds to the one-way time from the reference source to the diffractor $(i=s)$, and from the diffractor to the reference receiver $(i=g)$.

The moveout of point-like scatterers contains no information on the interface geometry, but is purely due to propagation from the scatterer to the receivers, which makes the multicoverage diffraction response inherently redundant and useful for velocity inversion (e. g. Bauer et al., 2016, 2017). Since every ray segment is normal to the diffracting structure, the finite-offset wavefront measurements at source and receiver can be identified with their NIP wave counterparts defined in the zero-offset section at the spatial locations of source and receiver (compare Figure 7). As illustrated on the right of Figure 8, the full prestack diffraction traveltime response can be fully decomposed into zero-offset NMO measurements

$$
t_{\mathrm{diff}}\left(\Delta x_{s}, \Delta x_{g}\right)=\frac{1}{2} \sum_{i=s, g}\left[t_{0}+\Delta \hat{t}(\rho=1, h=0)\right]_{i}
$$

where $t_{0}$ is the actual zero-offset two-way traveltime and $\Delta \hat{t}$ can be any of the generalized multi-parameter $\mathrm{NMO}$ expressions considered above where $\Delta x_{m}$ is set either to the relative 
finite-offset source $(i=s)$ or receiver $(i=g)$ displacement $\Delta x_{i}=x_{i}-x_{0 i}$.

For the above mentioned reasons, the moveout of a point diffractor in the shot gather is equivalent to the moveout of a passive seismic source located at the diffractor position

$$
\Delta t_{\mathrm{pass}}=\frac{\hat{R}_{g}-\hat{R}_{0 g}}{\hat{v}},
$$

with $\hat{R}_{g}$ and $\hat{R}_{0 g}$ defined according to (32) and (33). Again, depending on the choice of the auxiliary medium, the hatted attributes in the suggested wavefront formulation of the diffraction and the passive event moveout either reduce to effective or projected quantities. For a zero-offset reference ray the source and receiver wavefronts and their attributes coincide and we arrive at the general CRP formula (29). Setting $\hat{\alpha}=0$, we arrive either at the conventional Kirchhoff time migration operator in the effective medium, or its time-shifted projection analog (e.g. Schwarz et al., 2014). So the generalized multifocusing, implicit CRS and nonhyperbolic CRS expressions naturally contain the conventionally used time migration operators.

\section{IMPLICATIONS AND APPLICATIONS}

\section{Unification and multi-NMO analysis}

At this point, we would like to emphasize the special role of the multifocusing moveout, which, in contrast to all other presented methods like hyperbolic CRS and implicit CRS, was originally formulated for the optical projection in the constant near-surface velocity medium and, therefore, like the shifted hyperbola by de Bazelaire (1988), relies on a time shift to account for heterogeneity. Without commenting on the auxiliary medium itself, Landa (2007) already stated that the multifocusing moveout correction does not suffer from moveout stretch (since it does not depend on the reference traveltime $t_{0}$ ) and reduces 
to the time-shifted hyperbola by de Bazelaire in the CMP gather, when the overburden consists of a horizontally layered system.

Since generalized multifocusing, the generalized implicit CRS, and the generalized nonhyperbolic CRS all reduce to exactly the same expressions for planar horizontal layering in the CMP gather or the increasingly important diffraction case, we consider them being of comparable accuracy. All three operators reduce to the exact solution for one single planar reflector (in the CMP gather) and for a point diffractor with a constant velocity background. This supports the finding that all higher-order expressions with the same number of degrees of freedom as the parabolic formula (3), i.e. hyperbolic CRS, multifocusing, implicit CRS, and nonhyperbolic CRS are exact for a certain subsurface model and can also be derived from an auxiliary straight-ray geometry. Please note that these approaches, in consequence, are confined by an asymptotic curve or surface (illustrated in Figures 1 and 5). The parabolic formula neither has two faces, like the higher-order expressions, nor does an asymptote exist and it happens to be never exact, no matter how simple the model is chosen.

Since all presented generalized moveouts, for small vicinities, i.e. up to second order, are equivalent to the parabolic formula (3), and due to the fact that the three double-square-root expressions are capable of handling low and high reflector curvatures equally well provided that lateral velocity changes are moderate, we consider the suggested generalized versions of multifocusing, implicit CRS, and nonhyperbolic CRS the most general NMO expressions.

[Figure 9 about here.]

To ensure realistic circumstances and sufficient applicability of the resulting conclusions, we choose the complex BP 2004 velocity benchmark dataset (Billette and Brandsberg-Dahl, 
2005) for a systematic quantitative comparison, since it contains all relevant geological features that can lead to higher-order effects in the traveltime moveout. Resulting from the presence of salt, as can be observed in the top of Figure 9, the velocity-shifted implicit CRS stack of the BP multi-coverage data shows pronounced and complex diffraction patterns in the left part and strong surface-related multiple reflections on the right.

[Figure 10 about here.]

[Figure 11 about here.]

To clearly emphasize the differences of the two faces of the suggested generalized operators, and to motivate some potential applications of their simultaneous use, we here confine ourselves to the comparison of the wavefront attributes $\alpha$ and $R_{\mathrm{NIP}}$, which turn out to either efficiently characterize diffractions or velocity changes in the model. The bottom of Figure 9 shows the angle estimate difference of the two versions of the implicit CRS operator on the left and the corresponding deviations in $R_{\mathrm{NIP}}$ on the right. As can be inferred from these deviation patterns, the angle differences are only non-negligible for events stemming from highly curved structures like the complex rugged top-of-salt present in the left side of the model, whereas differences in $R_{\mathrm{NIP}}$ indicate the general presence and strength of velocity variations in the overburden. The surface-related multiple reflections dominating the right part of Figure 9 are not affected by velocity changes in the subsurface and can therefore be clearly distinguished from the primary reflections whose rays were exposed to the heterogeneity of the shallow layers in the model.

Figure 10(a) and Figure 10(b) show two closeups of Figure 9 (indicated by frames) for hyperbolic CRS (CRS), multifocusing (MF), and implicit CRS (iCRS). One can observe the 
strong systematics in the attribute deviations, which, for the case of the double-square-root operators only show when a time-shifted version is compared with an expression shifted in velocity. The time-shifted face of the implicit CRS operator turns out to estimate the attributes absolutely equivalently to the multifocusing formulation, which only appears in time-shifted notation in existing literature (e.g., Gelchinsky et al., 1999a,b). In accordance, the estimate differences vanish, when we compare the effective medium version of implicit CRS, which was suggested by Schwarz et al. (2014), with the new effective-medium (velocityshifted) formulation of multifocusing. The same behavior is also revealed for the hyperbolic CRS operator, indicating that the generalized operators all have the potential to provide additional insight into the character and physical origin of a recorded event. As is illustrated in Figure 11, when expressed in the same auxiliary medium, an attribute comparison of the hyperbolic CRS with e.g. the double-square-root implicit CRS operator likewise reveals a systematic deviation pattern for diffractions, which again suggests that the correct use of multiple NMO approximations can add value to the conventional processing chain.

\section{Improved diffraction filters}

[Figure 12 about here.]

In all considered examples the emergence angle and coherence differences between CRS and implicit CRS and between the dual descriptions of the implicit CRS were most pronounced for events stemming from highly curved subsurface features like diffracting structures, whereas the corresponding differences vanished for reflection events connected to moderately curved or nearly planar features in the respective models. Dell and Gajewski (2011) suggested to use an exponential weight of the difference between the normal and the 
NIP wave radius to efficiently discriminate reflection and diffraction events in the data

$$
w_{\text {Dell }}=\exp \left(-\frac{|1-\rho|}{|1+\rho|}\right),
$$

where $\rho$ is the dimensionless ratio of $R_{\mathrm{NIP}}$ and $R_{\mathrm{N}}$ (see equation (9)). Due to the fact that $w_{\text {Dell }}$ takes values between 0 and 1 , a threshold can be chosen to separate diffracted from reflected energy (Dell and Gajewski, 2011). Since the difference in the emergence angle estimation of the two versions of the implicit CRS operator appeared to be a very consistent and reliable diffraction indicator throughout the presented investigations, we suggest the following angle-based alternative to the conventional weight

$$
w_{\text {angle }}=1-\exp \left[-\left(\alpha_{v}^{2}-\alpha_{t}^{2}\right)\right],
$$

where $\alpha_{v}$ and $\alpha_{t}$ denote the emergence angle estimated with the velocity-shifted and the time-shifted version of the implicit CRS moveout, respectively. Figure 12 shows the corresponding diffraction weights, defined according to expressions (36) and (37), for the BP dataset. While the conventional weight by Dell and Gajewski (2011), estimated only with the velocity-shifted implicit CRS operator, generally provides a good discrimination between reflection and diffraction events especially in the vicinity of the diffractions' apices, the separation potential decreases noticeably for the steep flanks of the diffractions. In addition, reflections are still addressed with overall comparably large weights, which may lead to insufficient separation for a poorly user-chosen threshold. The angle-based alternative weight displayed in the center of Figure 12, in contrast proves to be very discriminative and, due to the fact that it utilizes attribute differences rather than similarities, as in the conventional approach, shows its strength for the steep diffraction flanks, where the conventional weight loses accuracy and reliability. Therefore, a combination of both weights (shown on the right in Figure 12) uses the advantages of both approaches. 


\section{Moveout stretch}

[Figure 13 about here.]

As indicated before, the choice of the auxiliary medium directly implies the choice of a specific mechanism to account for heterogeneity. Due to the fact that the effective medium moveouts all have in common that they directly depend on the zero-offset reference traveltime $t_{0}$, moveout stretch can be observed for all these approaches and also shows for data modeled in a hypothetical medium of perfectly constant velocity (see, e.g., Perroud and Tygel, 2004). For the sake of simplicity, we demonstrate this behavior for the hyperbolic CMP moveout (19) only, but we would like to stress that the presented findings also apply to the more general multi-dimensional NMO approaches. Figures 13(a) and 13(b) show isomoveout curves, i.e. curves of constant moveout, for the velocity-shifted CMP hyperbola and its time-shifted counterpart for a fixed finite-offset. Presented are five different constant moveouts with the shifts in velocity and in time as functions of the reference traveltime $t_{0}$. By comparing 13(a) and 13(b) we observe that in order to describe one particular moveout for different reference traveltimes $t_{0}$, the shift in velocity needs to be changed, whereas timeshifted moveouts remain constant for the whole range of reference traveltimes, and, therefore do not depend on $t_{0}$. This implies that in order to describe the moveout as a constant along the recorded seismic wavelet, the velocity shift needs to be changed in accordance with the operator's iso-moveout curve.

[Figure 14 about here.]

In consequence, the intuitive choice of a constant velocity shift for one event results in neglection of the slope of the velocity-shift iso-moveout curve along the wavelet, which leads 
to over-corrections for some parts and under-corrections for others, eventually causing an undesired increase of the signal period at higher offsets. As may be concluded from Figure 13(a), this effect is generally most pronounced at small times $t_{0}$, whereas it becomes almost negligible for higher values of $t_{0}$. As can also be inferred from Figure 13(b), the time-shifted moveouts do not show this dependency and the intuitive choice of a constant time-shift for a single event leaves the frequency content unchanged. Figure 14(a) and Figure 14(b) demonstrate this with two simple data examples, where the time-shifted and the velocity-shifted moveout corrections are applied to a series of equidistant horizontal reflectors in a constant velocity background and different events with the same moveout shifted in time. In both cases, when either a constant shifted velocity or shifted reference time is chosen for NMO correction, one can observe the effect of wavelet stretch exclusively for the velocity shift approach, whereas this phenomenon never shows for the time shift mechanism. Figure 15 illustrates that the previous findings likewise apply to the multidimensional NMO correction of the shallow waterbottom reflection of the BP velocity benchmark dataset, where a constant velocity shift results in significant wavelet stretch even for moderate offsets, while a constant shift of the reference time preserves temporal resolution over all considered offsets.

[Figure 15 about here.]

\section{Passive event localization}

In the context of NMO correction we have found that the undesired effect of moveout stretch is an inherent property of the velocity-shifted representation of each operator, whereas wavelets remain undistorted for the time-shift mechanism. In this final part of the paper, we seek to support the notion that both versions of each operator may not only be jointly 
utilized, but that the velocity-shift mechanism's characteristic dependence on the reference time $t_{0}$ is also advantageous for certain applications. In the context of passive seismic monitoring or earthquake seismology, the localization of the unknown source of a seismic event is a fundamental problem and may be approached with the data-driven method of diffraction stacking (Zhebel et al., 2011). For confining the source's horizontal position, description of moveout is sufficient and the reference time, according to the time shift mechanism, may be chosen arbitrarily. To achieve a localization in time, i.e. to estimate the source's time of excitation, however, only the effective medium approach is suited, since the velocity-shifted moveout directly depends on the true reference time $t_{0}$. This means that in order to allow for a localization in time, both, the reference time $t_{0}$ and the shifted velocity $v_{\text {shift }}$ (linked to a corresponding time shift through equation (12)) need to be estimated. To gain stability and decrease computational demands, Schwarz et al. (2016) suggested to first extract the local event slope using a first-order approximation, then optimize the shifted reference time $t_{\text {shift }}$ and, finally, estimate the true propagation time $t_{0}$ in a fitting procedure. Following this strategy, they made use of both versions of the dual passive event moveout (35) in local coherence analysis to successfully approximate the unknown hypocentral times of three spatially separated passive events. In addition, the estimated attributes could then be fed to wavefront tomography (Duveneck, 2004) leading to a reliable depth localization and joint velocity inversion without incorporating any a priori information besides the nearsurface velocity.

\section{CONCLUSIONS}

We have shown that conventional and multi-parameter NMO expressions can both be conveniently derived from the same simple geometry of locally circular emerging wavefronts. 
We argue that all moveout operators that provide the exact solution to a certain subsurface model are non-parabolic and may be represented geometrically in an auxiliary medium of constant velocity. While depth methods are known to be superior over time imaging in the presence of strong lateral medium complexity, in NMO analysis, effects of predominantly vertical heterogeneity are conventionally accounted for by shifting the constant velocity of the auxiliary medium. Alternatively, previous studies demonstrated, that a shift in the reference time can lead to a similar adaption of the traveltime moveout in the vicinity of the reference ray. Continuing the work of de Bazelaire in the late eighties, we have suggested generalized expressions of the classical CMP hyperbola, conventional CRS, and the double-square-root expressions of multifocusing, implicit CRS and nonhyperbolic CRS, which may be shifted either in velocity or in time. As a consequence, we observed that the two different versions of each operator show systematically different behavior in the presence of heterogeneity. This generalization revealed that the multifocusing moveout, in contrast to the other multi-parameter NMO expressions, was originally formulated for the problem's optical projection, where a time-shift accounts for heterogeneity. Comparison of double-square-root-based implicit CRS and multifocusing revealed that both operators are essentially equivalent, when the same auxiliary medium for both operators is considered. Application of both versions of an operator for the complex BP 2004 velocity benchmark dataset not only confirmed unification, but also revealed potential for diffraction or multiple identification. While time-shifted moveout corrections generally do not suffer from wavelet stretch, effective operators permit the estimation of the true reference traveltime, which paves the road for passive seismic applications. 


\section{ACKNOWLEDGMENTS}

We thank the Applied Seismics Group Hamburg, in particular Claudia Vanelle who reminded us of the pioneering work of Shah (1973), for continuous discussions. In addition, we gratefully acknowledge the helpful suggestions of John Etgen, Ian Jones, Hassan Masoomzadeh, and an anonymous reviewer. This work was partly financed by the sponsors of the Wave Inversion Technology (WIT) Consortium and the project 'Imaging steep structures with diffractions', which is funded by the German Ministry of Environment (BMU 0325363C). The first author is supported by a research fellowship of the German Research Foundation (DFG, SCHW 1870/1-1). 


\section{REFERENCES}

Bauer, A., B. Schwarz, and D. Gajewski, 2016, Enhancement of prestack diffraction data and attributes using a traveltime decomposition approach: Studia Geophysica et Geodaetica, 60, 471-486.

— 2017, Utilizing diffractions in wavefront tomography: Geophysics, 80, R65-R73.

Berkovitch, A., K. Deev, and E. Landa, 2011, How non-hyperbolic Multifocusing improves depth imaging: First Break, 27, 95-103.

Billette, F., and S. Brandsberg-Dahl, 2005, The 2004 BP Velocity Benchmark: Presented at the 67th EAGE Conference \& Exhibition.

Bortfeld, R., 1989, Geometrical ray theory: Rays and traveltimes in seismic systems (secondorder approximations of the traveltimes): Geophysics, 54, 342-349.

Castle, R. J., 1994, A theory of normal moveout: Geophysics, 59, 983-999.

Cruz, J., P. Hubral, M. Tygel, J. Schleicher, and G. Höcht, 2000, The common reflecting element (CRE) method revisited: Geophysics, 65, 979-993.

de Bazelaire, E., 1988, Normal moveout revisited - inhomogeneous media and curved interfaces: Geophysics, 53, 143-157.

Dell, S., and D. Gajewski, 2011, Common-reflection-surface-based workflow for diffraction imaging: Geophysics, 76, S187-S195.

Deregowski, S., 1986, What is DMO?: First Break, 4, 7-24.

Duveneck, E., 2004, Velocity model estimation with data-derived wavefront attributes: Geophysics, 69, 265-274.

Fomel, S., and V. Grechka, 2001, Nonhyperbolic reflection moveout of P waves. An overview and comparison of reasons: CWP-372: Colorado School of Mines.

Fomel, S., and R. Kazinnik, 2013, Non-hyperbolic common reflection surface: Geophysical 
Prospecting, 61, 21-27.

Fomel, S., and A. Stovas, 2010, Generalized nonhyperbolic moveout approximation: Geophysics, 75, U9-U18.

Gelchinsky, B., A. Berkovitch, and S. Keydar, 1999a, Multifocusing homeomorphic imaging - part 1. Basic concepts and formulae: Journal of Applied Geophysics, 42, 229-242.

—, 1999b, Multifocusing homeomorphic imaging: Part 2. Multifold data set and multifocusing: Journal of Applied Geophysics, 42, 243-260.

Hertweck, T., J. Schleicher, and J. Mann, 2007, Data stacking beyond CMP: The Leading Edge, 26, 818-827.

Höcht, G., E. de Bazelaire, P. Majer, and P. Hubral, 1999, Seismics and optics: hyperbolae and curvatures: Journal of Applied Geophysics, 42, 261-281.

Hubral, P., 1983, Computing true amplitude reflections in a laterally inhomogeneous earth: Geophysics, 48, 1051-1062.

Hubral, P., T. Krey, and K. L. Larner, 1980, Interval velocities from seismic reflection time measurements: Society of Exploration Geophysicists.

Jäger, R., J. Mann, G. Höcht, and P. Hubral, 2001, Common-reflection-surface stack: Image and attributes: Geophysics, 66, 97-109.

Khaidukov, V., E. Landa, and T. J. Moser, 2004, Diffraction imaging by focusing-defocusing: An outlook on seismic superresolution: Geophysics, 69, 1478-1490.

Landa, E., 2007, Beyond conventional seismic imaging: European Association of Geoscientists and Engineers.

Landa, E., S. Keydar, and T. J. Moser, 2010, Multifocusing revisited - inhomogeneous media and curved interfaces: Geophysical Prospecting, 58, 925-938.

Levin, F. K., 1971, Apparent velocity from dipping interface reflections: Geophysics, 36, 
$510-516$.

Malovichko, A. A., 1978, A new representation of the traveltime curves of reflected waves in horizontally layered media: Applied Geophysics, 91, 47-53.

Mann, J., 2002, Extensions and Applications of the Common-Reflection-Surface Stack Method: PhD thesis, University of Karlsruhe.

Mayne, W. H., 1962, Common reflection point horizontal data stacking techniques: Geophysics, 27, 927-938.

Perroud, H., P. Hubral, and G. Höcht, 1999, Common-reflection-point stacking in laterally inhomogeneous media: Geophysical Prospecting, 47, 1-24.

Perroud, H., and M. Tygel, 2004, Nonstretch NMO: Geophysics, 69, 599-607.

Schleicher, J., M. Tygel, and P. Hubral, 1993, Parabolic and hyperbolic paraxial two-point traveltimes in 3D media: Geophysical Prospecting, 41, 495-513.

Schwarz, B., A. Bauer, and D. Gajewski, 2016, Passive seismic source localization via common-reflection-surface attributes: Studia Geophysica et Geodaetica, 60, 531-546.

Schwarz, B., and D. Gajewski, 2017, The two faces of NMO: The Leading Edge, 36, 512517.

Schwarz, B., C. Vanelle, D. Gajewski, and B. Kashtan, 2014, Curvatures and inhomogeneities: An improved common-reflection-surface approach: Geophysics, 79, S231-S240.

Shah, P. M., 1973, Use of wavefront curvature to relate seismic data with subsurface parameters: Geophysics, 38, 812-825.

Taner, M. T., and F. Koehler, 1969, Velocity spectra-digital computer derivation applications of velocity functions: Geophysics, 34, 859-881.

Tygel, M., L. T. Santos, and J. Schleicher, 1999, Multifocus moveout revisited: derivations and alternative expressions: Journal of Applied Geophysics, 42, 319-331. 
Ursin, B., 1982, Quadratic wavefront and traveltime approximations in inhomogeneous layered media with curved interfaces: Geophysics, 47, 1012-1021.

Červený, V., 2001, Seismic ray theory: Cambridge University Press.

Walda, J., B. Schwarz, and D. Gajewski, 2017, A competitive comparison of multiparameter stacking operators: Geophysics, 82, 1-46.

Zhebel, O., D. Gajewski, and C. Vanelle, 2011, Localization of seismic events in 3D media by diffraction stacking: Presented at the 73rd EAGE Conference \& Exhibition, European Association of Geoscientists and Engineers. 


\section{LIST OF FIGURES}

1 Two different ways to change the CMP moveout to account for heterogeneity: (a) Classical approach, where the velocity is perturbed. (b) Application of a shift $\delta t$ to the reference time $t_{0}$. Actual traveltime curves are in bold lines, their respective asymptotes are dotted. In both cases black indicates the perturbed moveout, whereas gray denotes the unperturbed moveout. . . . .

2 Illustration of (a) the normal-incidence-point (NIP) and (b) the normal (N) wavefront in a heterogeneous velocity model (Hubral, 1983). Both result from fictitious one-way experiments that replace the more complicated twoway ray geometry in the common-reflection-point gather and the zero-offset section, respectively. . . . . . . . . . . . . . . . .

3 In case of a single planar target reflector with constant velocity overburden (left), the wavefront curvature $R_{0}$ and the distance to the reflector coincide, while they differ for the heterogeneous case (right). The solid black lines represent the actual raypaths, whereas gray colors indicate the straight-ray optical analogue described in the time-shift approach. . . . . . . . . . .

4 In the paraxial vicinity of the reference location $x_{0}$, the emerging wavefront can be approximated by the segment of a circle. Its local tilt and curvature can be parameterized by the emergence angle $\alpha$ and the radius of curvature

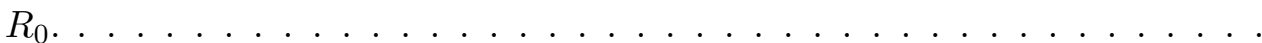

$5 \quad$ Velocity-shifted CRS moveout (a) and time-shifted CRS moveout (b). Like for the CMP stack, the perturbed moveout surfaces (solid grid) and their asymptotes (hollow grid) are shown in black. . . . . . . . . . . . . .

6 Illustration of the circular wavefront geometry that can be used to derive the double-square-root expressions of multifocusing (a), the implicit CRS (b), and the nonhyperbolic CRS moveout $(\mathrm{c}) . \ldots \ldots \ldots$

7 In the reflection case (a), the normal-incidence-point (NIP) wave attributes are defined at the emergence location of the normal ray only. For diffractions (b), every ray is a normal ray and the finite-offset and zero-offset wavefront measurements are inherently redundant (e.g. Bauer et al., 2016). . . . . . .

8 Redundancy of the finite-offset emergence angle at the source for a diffractor in a gradient background (left) and the decomposition of accurate finiteoffset diffraction traveltime operators (defined at the reference coordinates $\left.\left(x_{0 s}, x_{0 g}\right)\right)$ into zero-offset operators defined at $x_{0 s}$ and $x_{0 g}$, respectively (right). The whwite dotted line on the left indicates the common-shot gather. 48

9 The velocity-shifted implicit CRS stack of the 2004 BP velocity benchmark data (top) and the respective differences of the attributes $\alpha$ shown on the left and $R_{\mathrm{NIP}}$ shown in the right part of the section (bottom), estimated with the time-shifted and velocity shifted version of the implicit CRS, respectively. The black frames indicate closeups shown in Figures 10(a), 10(b), and 11. . 
10 Closeup of the difference section of the emergence angle $\alpha$ (a) and the NIP wavefront radius $R_{\mathrm{NIP}}$ (b) estimated with the two versions of conventional CRS (right), implicit CRS (iCRS), and multifocusing (MF). . . . . . . .

11 Closeup of the difference sections resulting from the use of the velocity-shifted versions of hyperbolic CRS and the double-square-root implicit CRS operator (compare Figure 9). . . . . . . . . . . . . . . . . . . . . .

12 Closeup of the conventional $\rho$-based diffraction weight by Dell and Gajewski (2011) (left), the new weight based on the systematic angle estimation differences of the two versions of the generalized implicit CRS moveout (middle) and their combination (right) . . . . . . . . . . . . . . .

13 Iso-moveout curves for the velocity-shifted classical NMO hyperbola (a) and the time-shifted hyperbola by de Bazelaire (b) for a fixed finite-offset. Although of higher dimensionality, similar dependencies also apply for the surface-based hyperbolic CRS, multifocusing, implicit CRS, and the nonhyperbolic CRS approximations and their subsets. . . . . . . . . . .

14 Time and velocity-shifted moveout correction applied (a) to a set of planar reflectors in a constant-velocity background, and (b) to time-shifted events with constant moveout. Next to the corrected gathers, the respective semblance panels are shown (the dotted line indicates the constant NMO parameter used for the correction). . . . . . . . . . . . . . . . . . . . .

15 Constant velocity shift (top) and constant time shift (bottom) NMO correction of the shallow waterbottom reflection of the BP velocity benchmark dataset, performed in a multi-dimensional supergather with the hyperbolic CRS approximation. The displayed supergather corresponds to the CMP range $10,001-10,050$ (compare Figure 9$) \ldots \ldots \ldots$ 


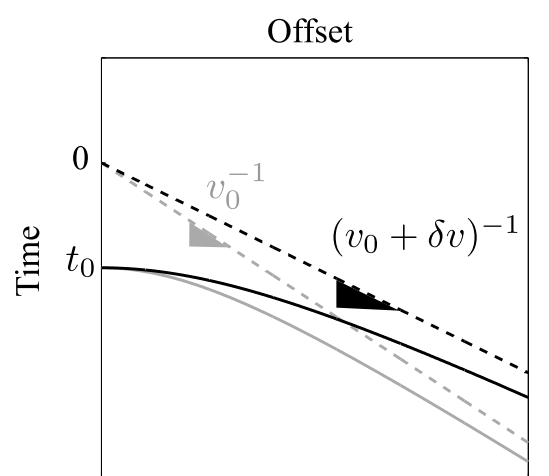

(a)

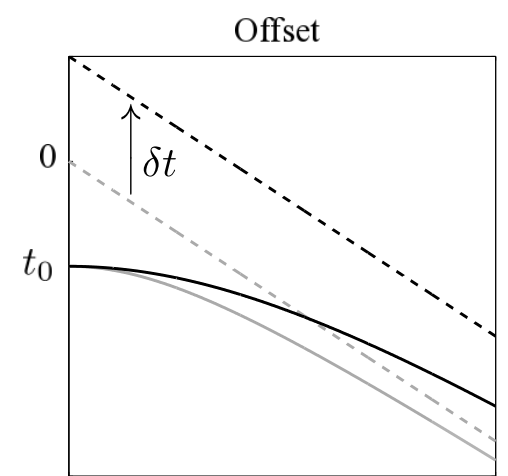

(b)

Figure 1: Two different ways to change the CMP moveout to account for heterogeneity: (a) Classical approach, where the velocity is perturbed. (b) Application of a shift $\delta t$ to the reference time $t_{0}$. Actual traveltime curves are in bold lines, their respective asymptotes are dotted. In both cases black indicates the perturbed moveout, whereas gray denotes the unperturbed moveout. 


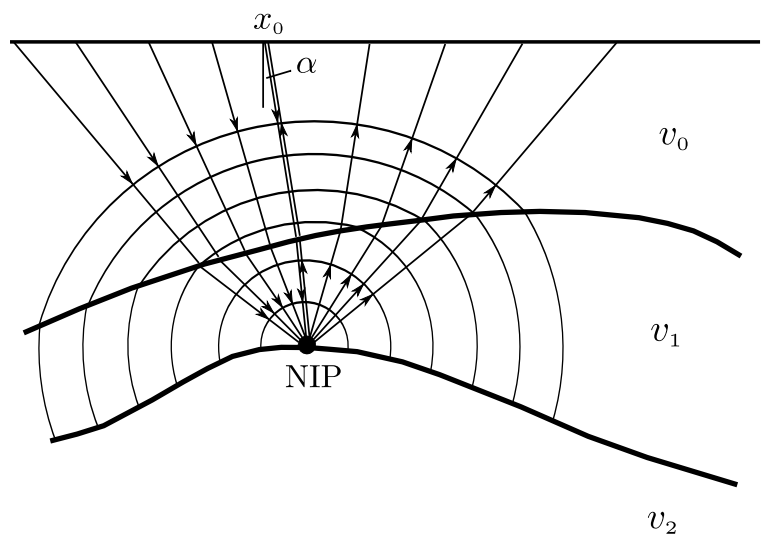

(a)

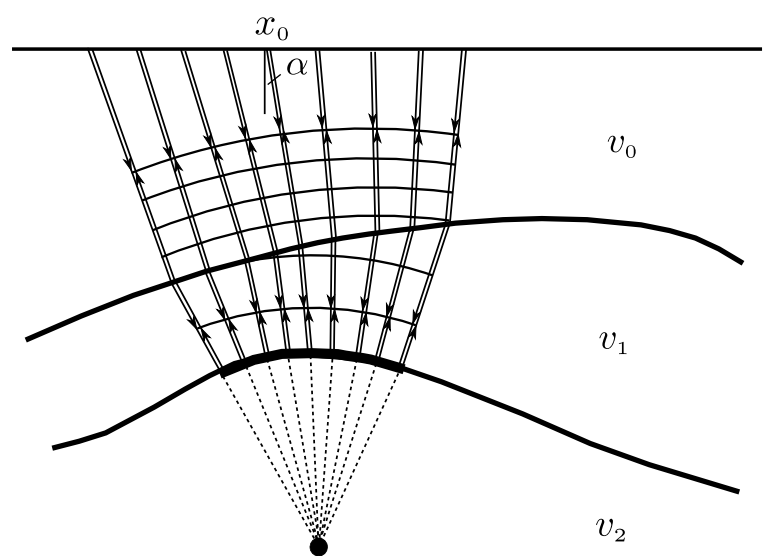

(b)

Figure 2: Illustration of (a) the normal-incidence-point (NIP) and (b) the normal (N) wavefront in a heterogeneous velocity model (Hubral, 1983). Both result from fictitious oneway experiments that replace the more complicated two-way ray geometry in the commonreflection-point gather and the zero-offset section, respectively. 


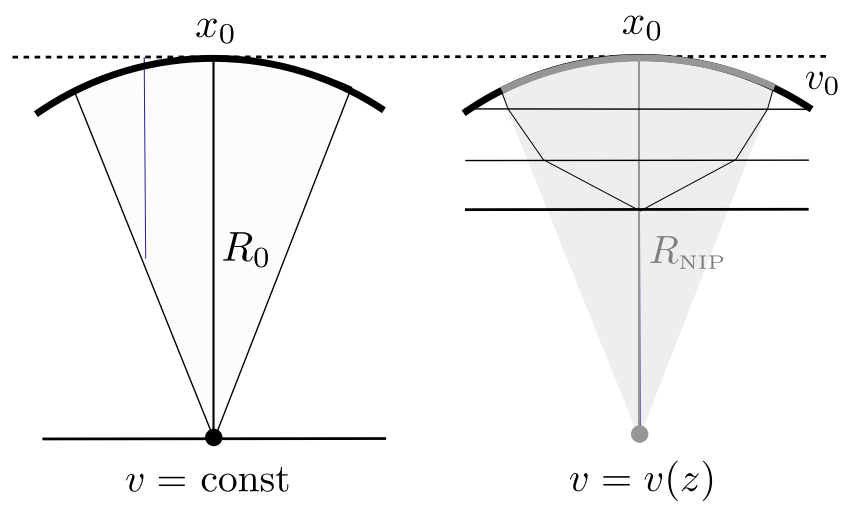

Figure 3: In case of a single planar target reflector with constant velocity overburden (left), the wavefront curvature $R_{0}$ and the distance to the reflector coincide, while they differ for the heterogeneous case (right). The solid black lines represent the actual raypaths, whereas gray colors indicate the straight-ray optical analogue described in the time-shift approach. 


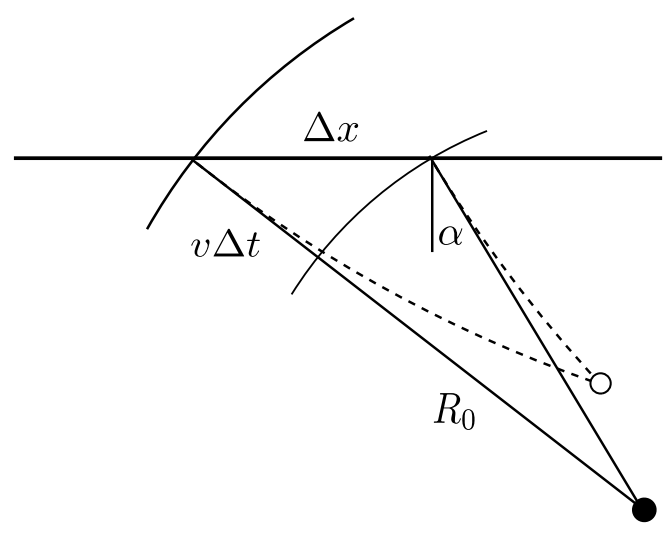

Figure 4: In the paraxial vicinity of the reference location $x_{0}$, the emerging wavefront can be approximated by the segment of a circle. Its local tilt and curvature can be parameterized by the emergence angle $\alpha$ and the radius of curvature $R_{0}$. 

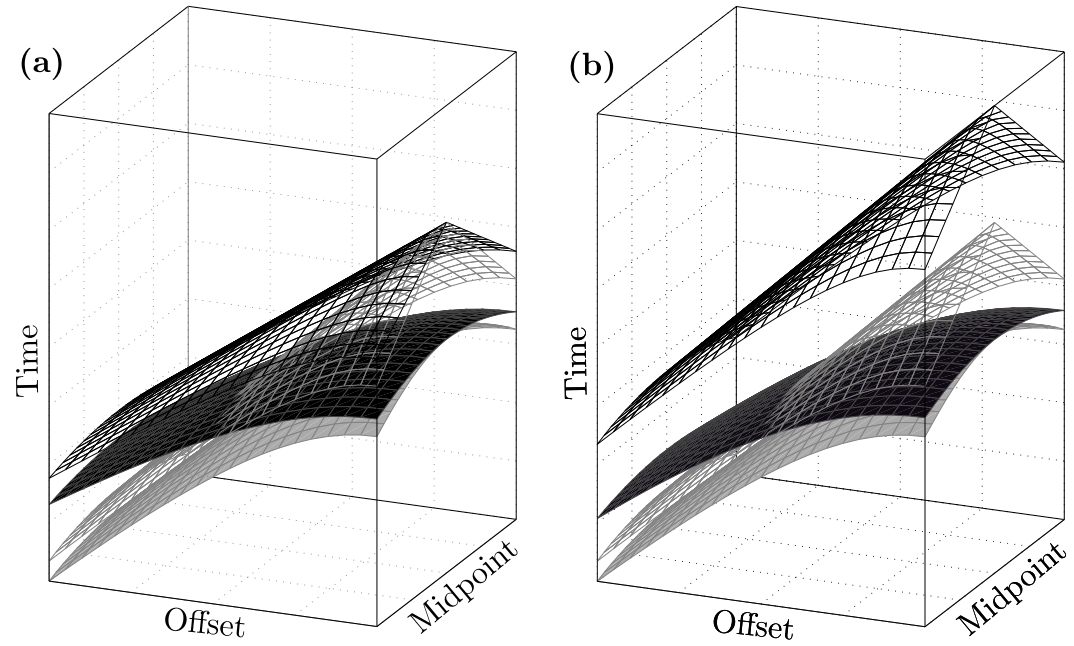

Figure 5: Velocity-shifted CRS moveout (a) and time-shifted CRS moveout (b). Like for the CMP stack, the perturbed moveout surfaces (solid grid) and their asymptotes (hollow grid) are shown in black. 

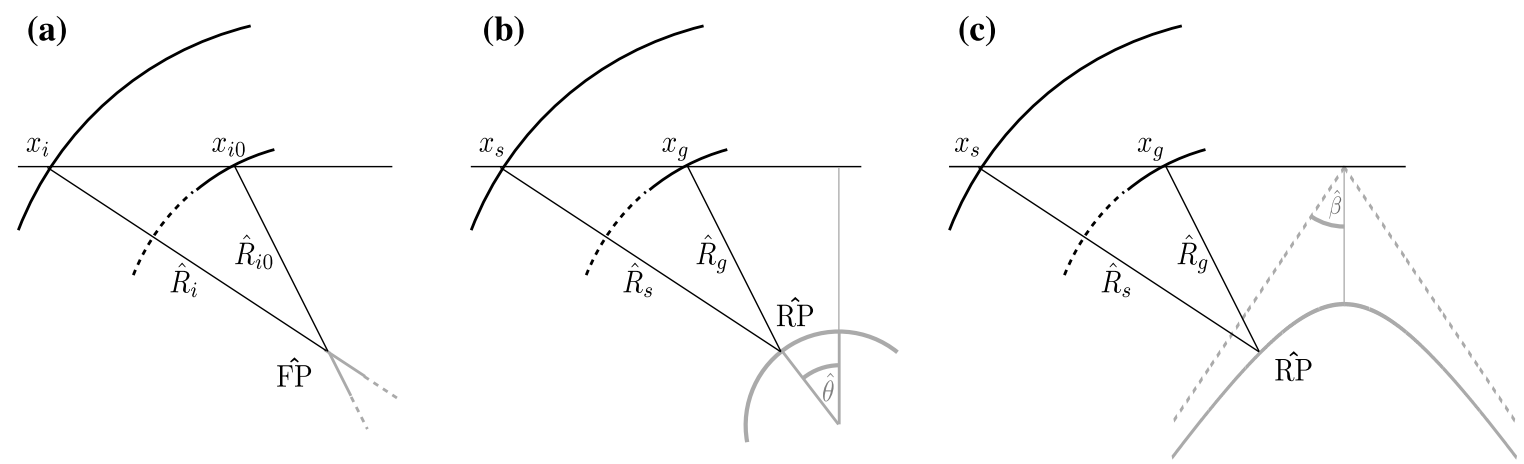

Figure 6: Illustration of the circular wavefront geometry that can be used to derive the double-square-root expressions of multifocusing (a), the implicit CRS (b), and the nonhyperbolic CRS moveout (c). 

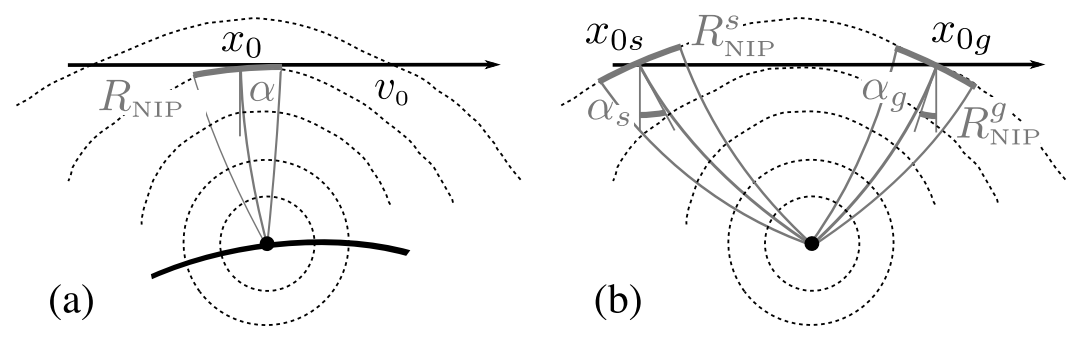

Figure 7: In the reflection case (a), the normal-incidence-point (NIP) wave attributes are defined at the emergence location of the normal ray only. For diffractions (b), every ray is a normal ray and the finite-offset and zero-offset wavefront measurements are inherently redundant (e.g. Bauer et al., 2016). 

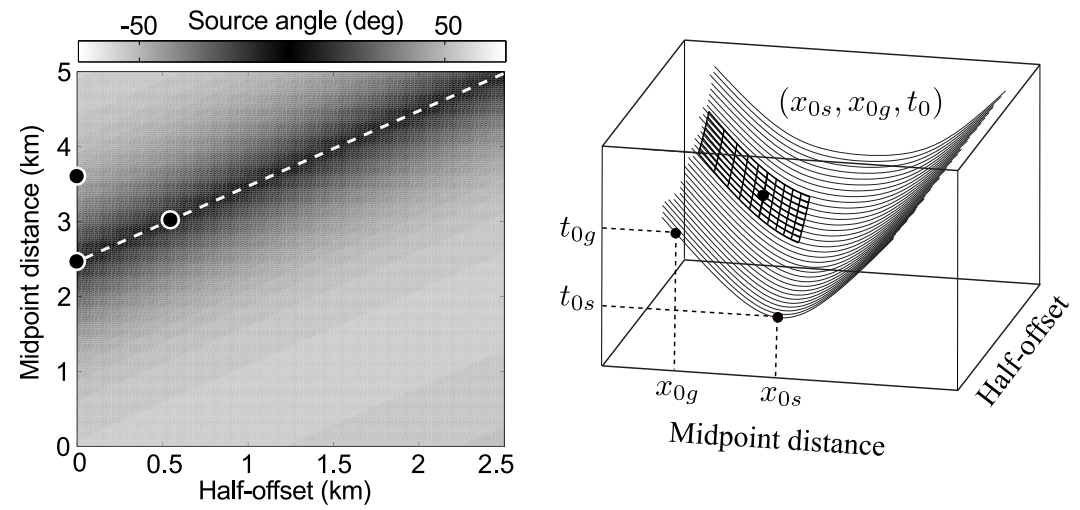

Figure 8: Redundancy of the finite-offset emergence angle at the source for a diffractor in a gradient background (left) and the decomposition of accurate finite-offset diffraction traveltime operators (defined at the reference coordinates $\left(x_{0 s}, x_{0 g}\right)$ ) into zero-offset operators defined at $x_{0 s}$ and $x_{0 g}$, respectively (right). The whwite dotted line on the left indicates the common-shot gather. 


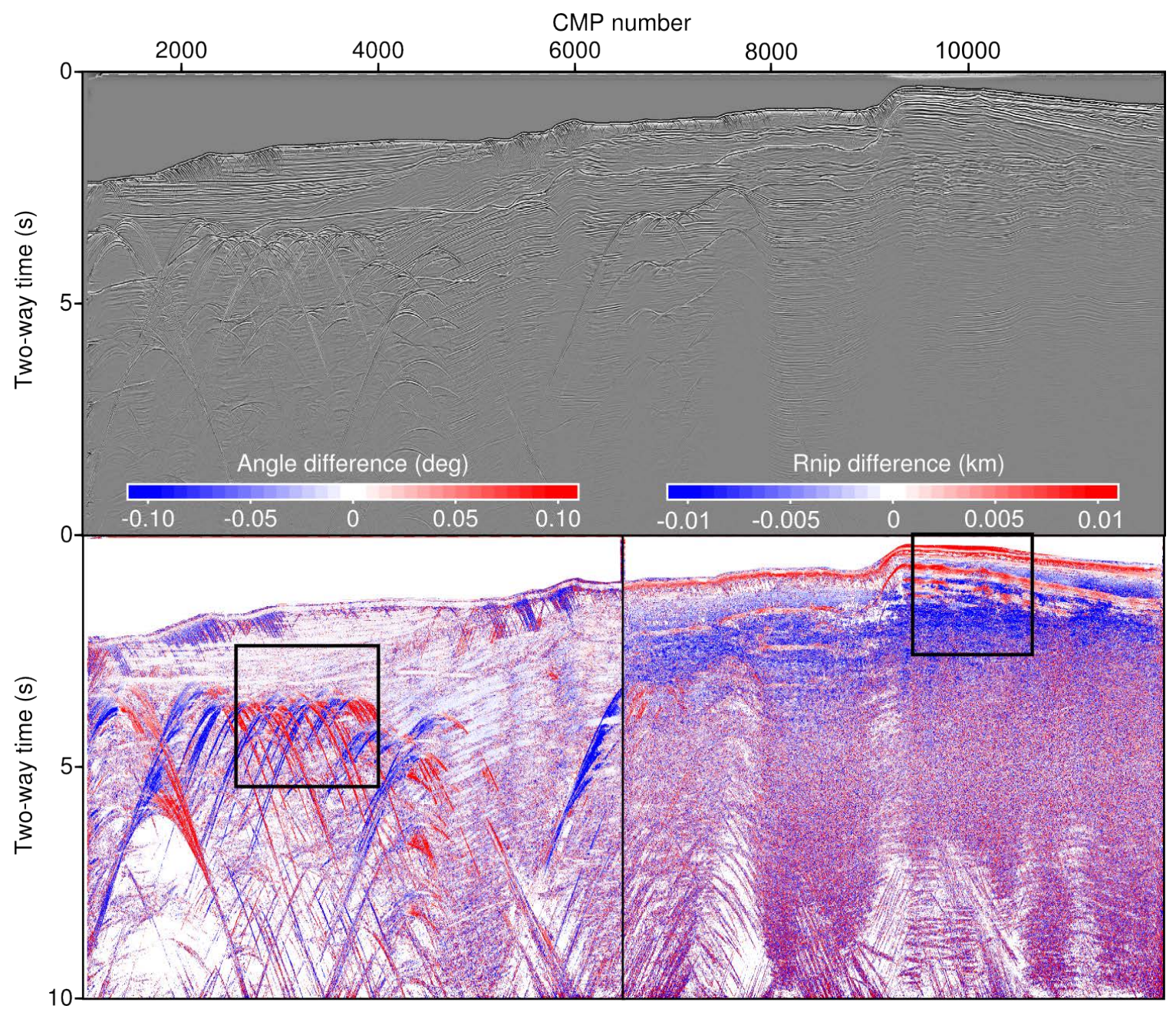

Figure 9: The velocity-shifted implicit CRS stack of the 2004 BP velocity benchmark data (top) and the respective differences of the attributes $\alpha$ shown on the left and $R_{\mathrm{NIP}}$ shown in the right part of the section (bottom), estimated with the time-shifted and velocity shifted version of the implicit CRS, respectively. The black frames indicate closeups shown in Figures 10(a), 10(b), and 11. 

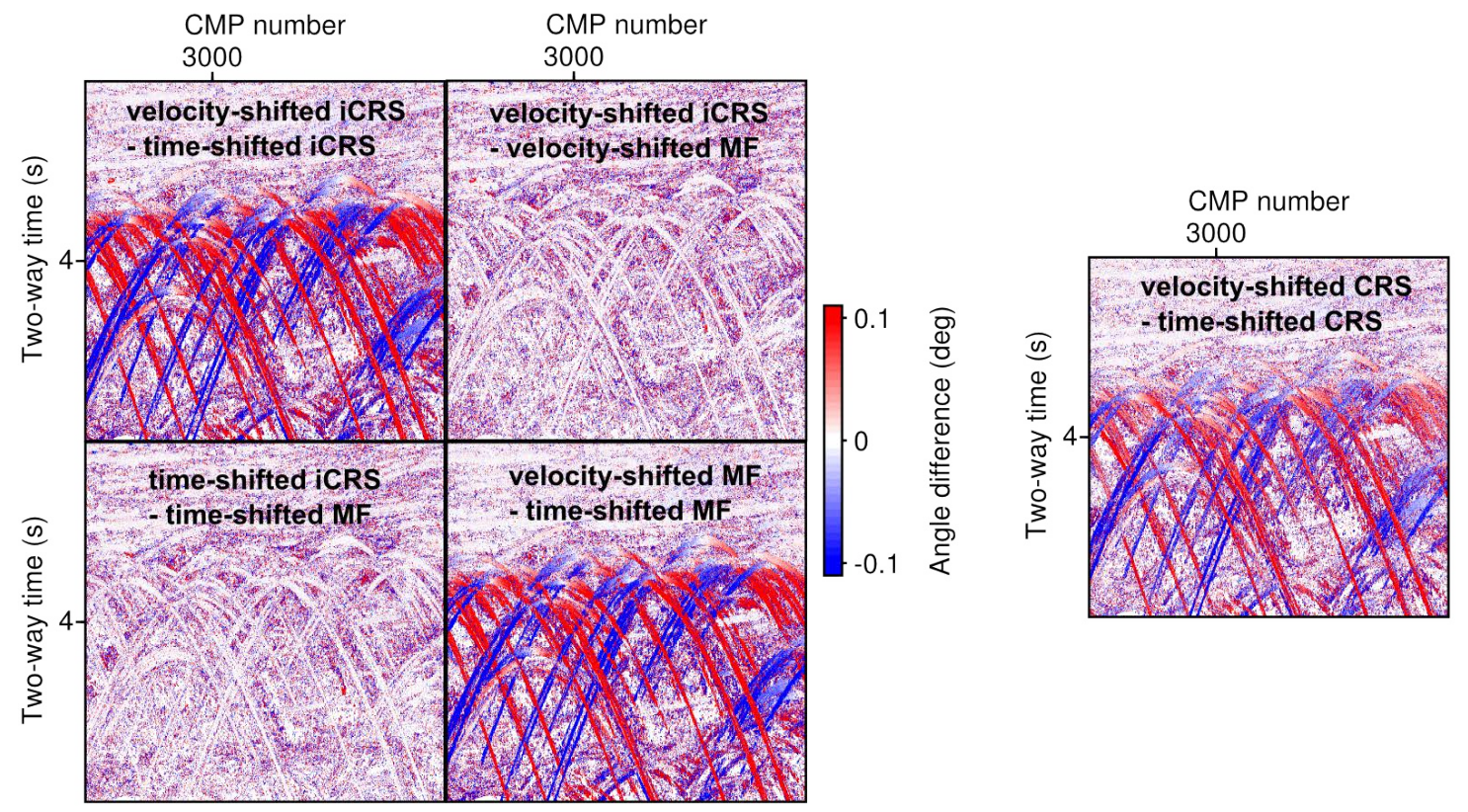

(a)
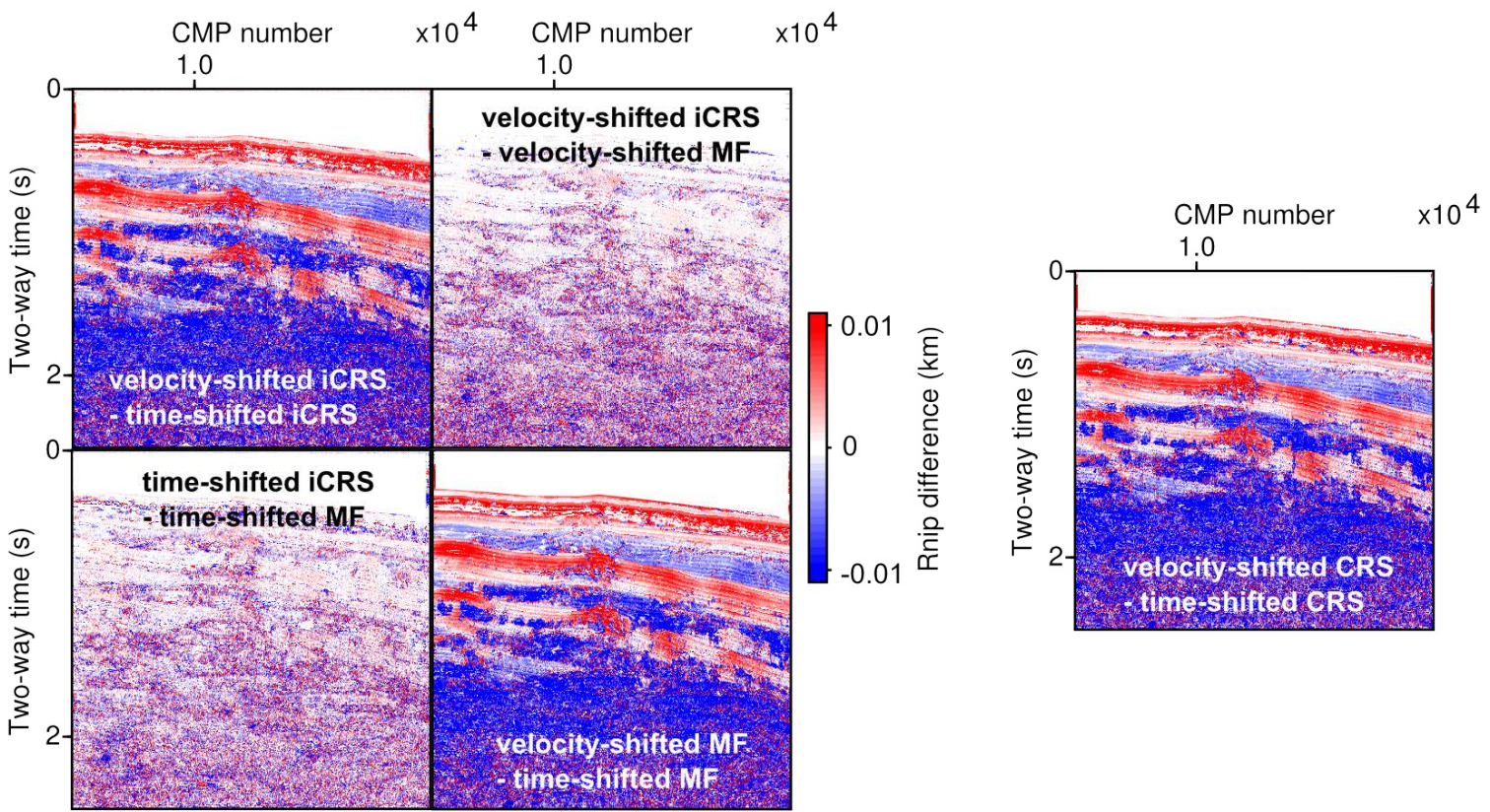

(b)

Figure 10: Closeup of the difference section of the emergence angle $\alpha$ (a) and the NIP wavefront radius $R_{\mathrm{NIP}}$ (b) estimated with the two versions of conventional CRS (right), implicit CRS (iCRS), and multifocusing (MF). 


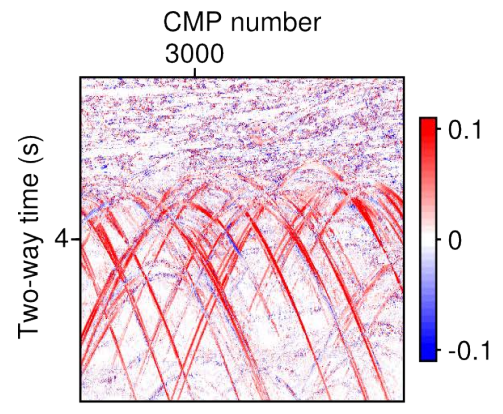

Semblance difference
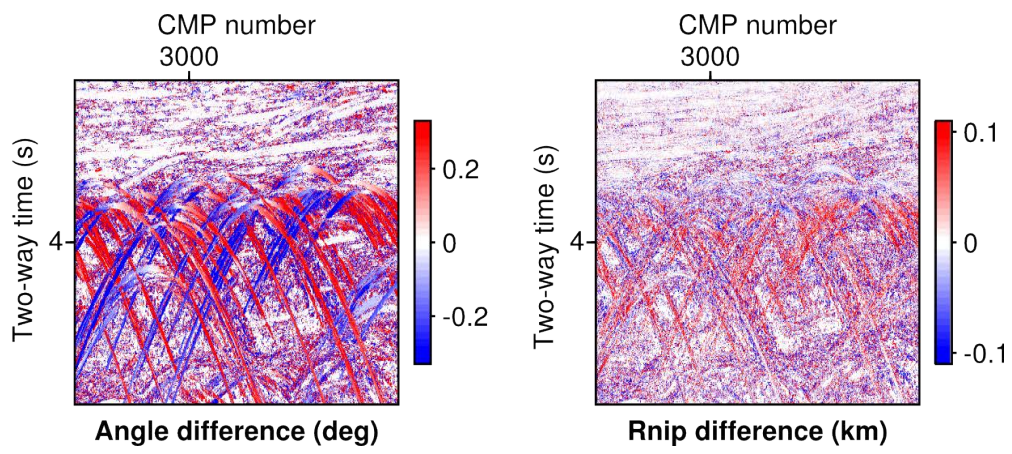

Figure 11: Closeup of the difference sections resulting from the use of the velocity-shifted versions of hyperbolic CRS and the double-square-root implicit CRS operator (compare Figure 9). 


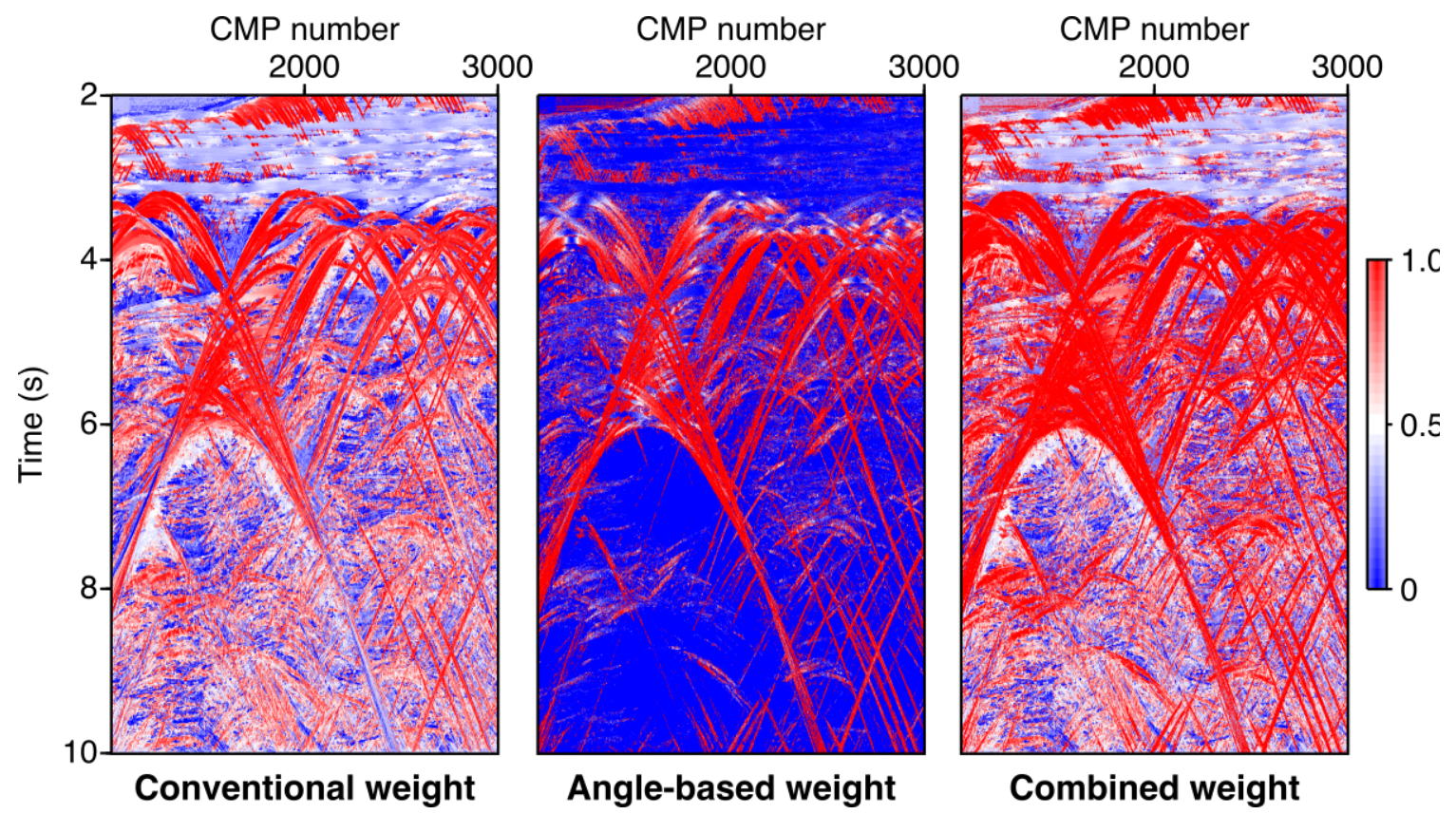

Figure 12: Closeup of the conventional $\rho$-based diffraction weight by Dell and Gajewski (2011) (left), the new weight based on the systematic angle estimation differences of the two versions of the generalized implicit CRS moveout (middle) and their combination (right). 


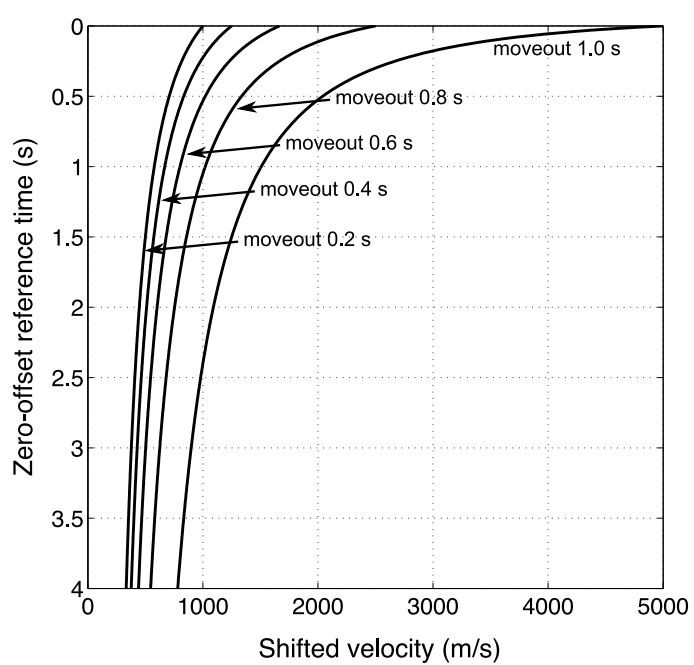

(a)

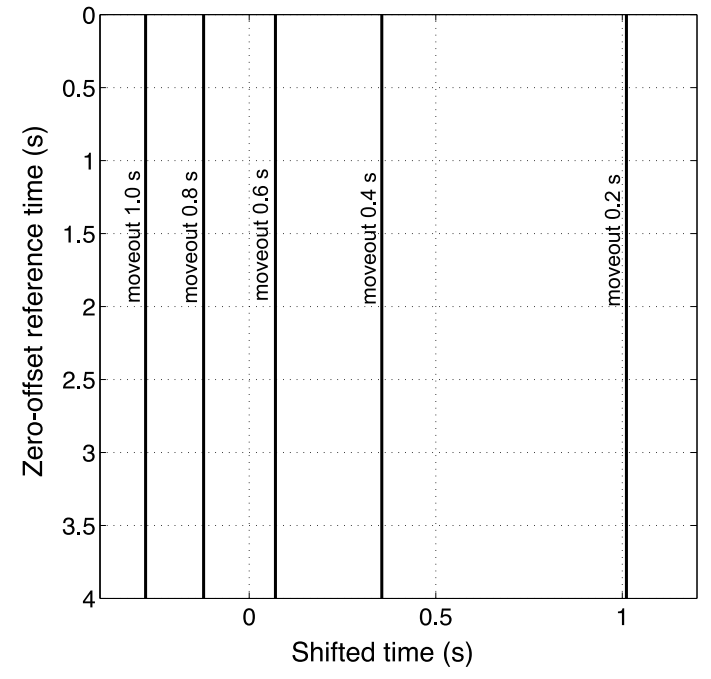

(b)

Figure 13: Iso-moveout curves for the velocity-shifted classical NMO hyperbola (a) and the time-shifted hyperbola by de Bazelaire (b) for a fixed finite-offset. Although of higher dimensionality, similar dependencies also apply for the surface-based hyperbolic CRS, multifocusing, implicit CRS, and the nonhyperbolic CRS approximations and their subsets. 


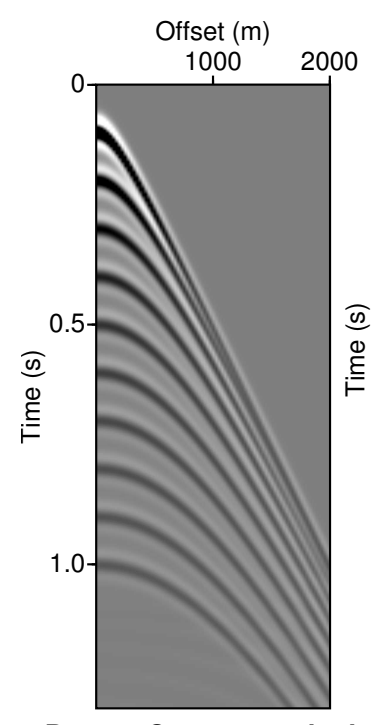

Data 1: Constant velocity
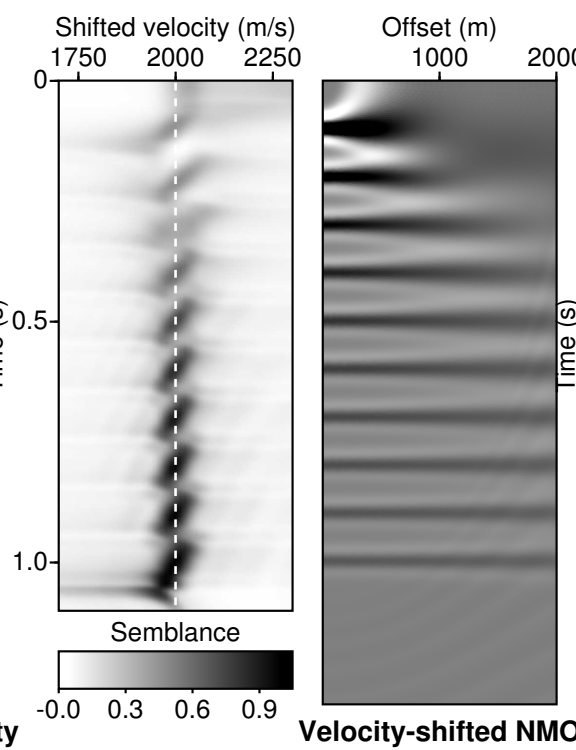

(a)

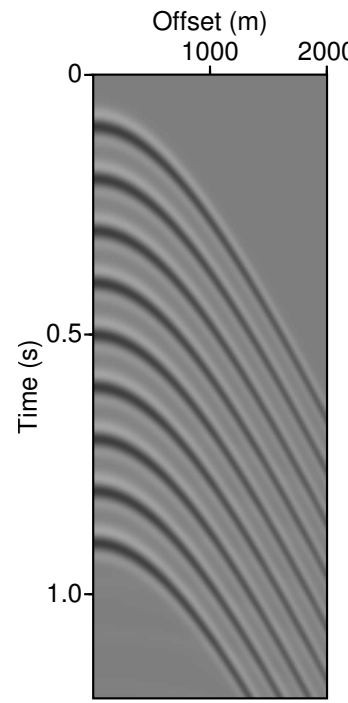

Data 2: Constant moveout
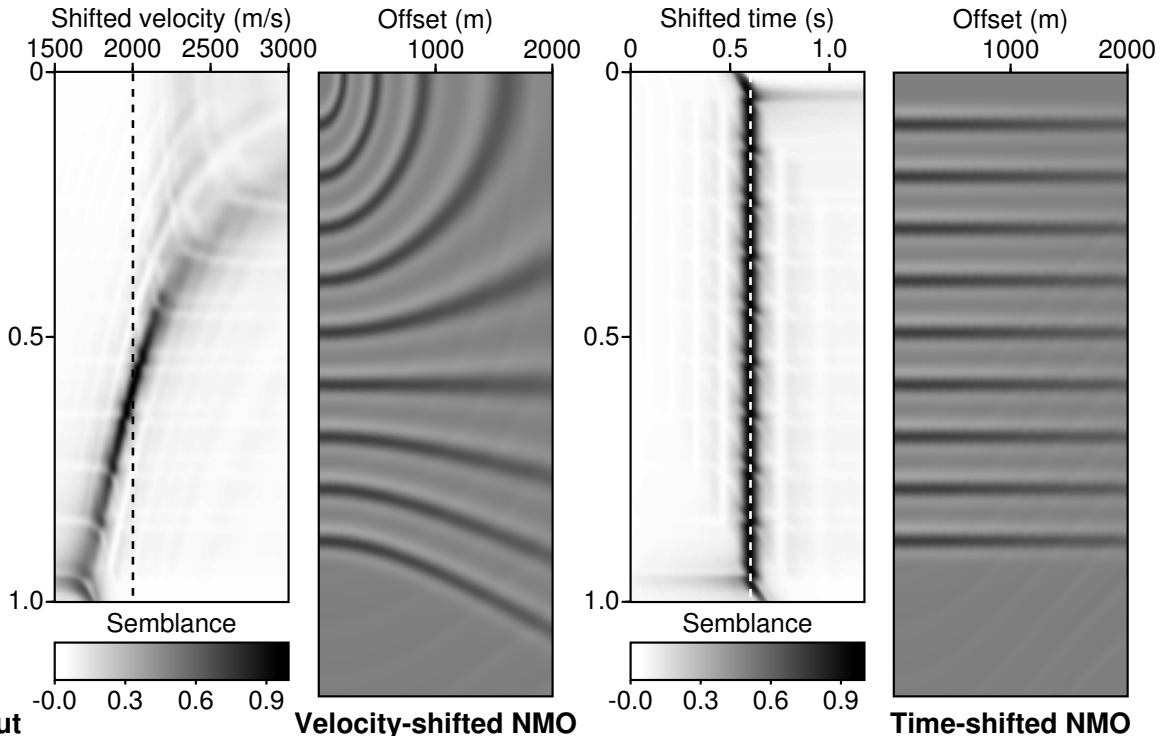

(b)

Figure 14: Time and velocity-shifted moveout correction applied (a) to a set of planar reflectors in a constant-velocity background, and (b) to time-shifted events with constant moveout. Next to the corrected gathers, the respective semblance panels are shown (the dotted line indicates the constant NMO parameter used for the correction). 

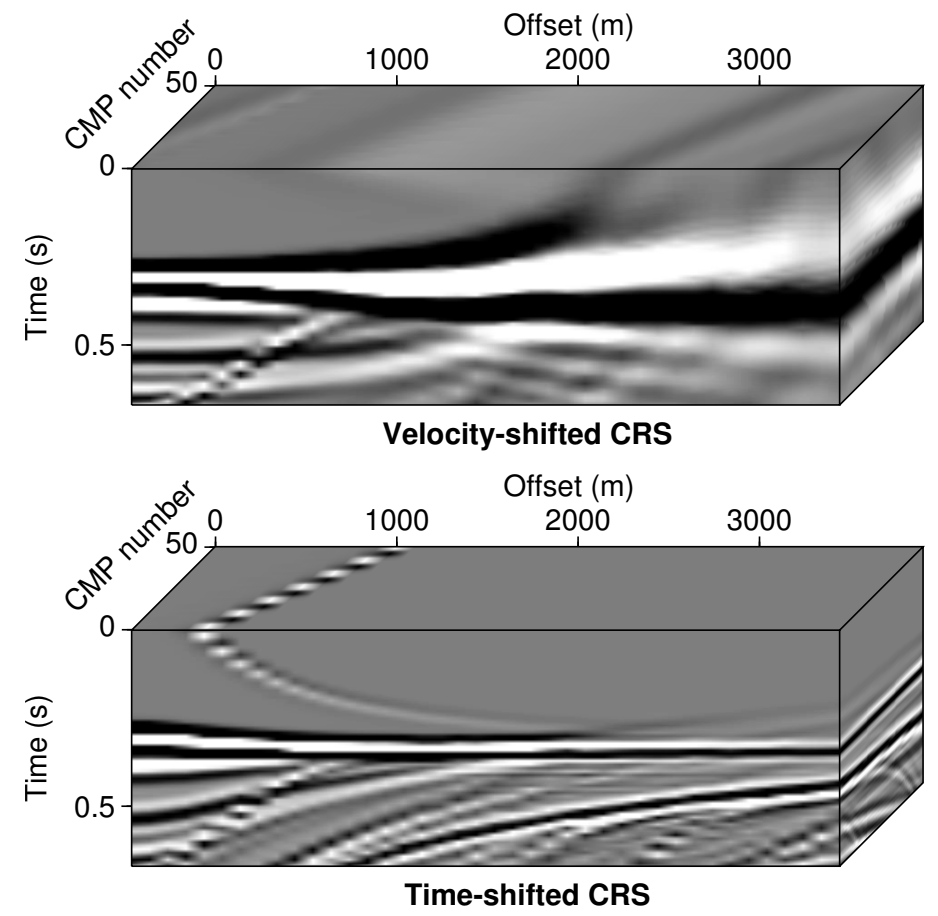

Figure 15: Constant velocity shift (top) and constant time shift (bottom) NMO correction of the shallow waterbottom reflection of the BP velocity benchmark dataset, performed in a multi-dimensional supergather with the hyperbolic CRS approximation. The displayed supergather corresponds to the CMP range 10,001 - 10,050 (compare Figure 9). 